\title{
The Sloan Digital Sky Survey Quasar Lens Search. V. Final Catalog from the Seventh Data Release
}

\author{
Naohisa Inada, ${ }^{1,2}$ Masamune Oguri, ${ }^{3,4}$ Min-Su Shin, ${ }^{5,6}$ Issha Kayo, ${ }^{3,7}$ Michael A. Strauss, ${ }^{6}$ \\ Tomoki Morokuma, ${ }^{8}$ Cristian E. Rusu, ${ }^{9,10}$ Masataka Fukugita, ${ }^{3,11}$ Christopher S. \\ Kochanek, ${ }^{12}$ Gordon T. Richards, ${ }^{13}$ Donald P. Schneider, ${ }^{14,15}$ Donald G. York, ${ }^{16,17}$ Neta A. \\ Bahcall, ${ }^{6}$ Joshua A. Frieman, ${ }^{16,18,19}$ Patrick B. Hall, ${ }^{20}$ and Richard L. White ${ }^{21}$
}




\footnotetext{
ABSTRACT

${ }^{1}$ Department of Physics, Nara National College of Technology, Yamatokohriyama, Nara 639-1080, Japan.

${ }^{2}$ Research Center for the Early Universe, School of Science, University of Tokyo, Bunkyo-ku, Tokyo 113-0033, Japan.

${ }^{3}$ Institute for the Physics and Mathematics of the Universe, The University of Tokyo, 5-1-5 Kashiwa-noha, Kashiwa, Chiba 277-8568, Japan.

${ }^{4}$ Division of Theoretical Astronomy, National Astronomical Observatory, 2-21-1, Osawa, Mitaka, Tokyo 181-8588, Japan.

${ }^{5}$ Department of Astronomy, University of Michigan, 500 Church Street, Ann Arbor, MI 48109-1042 USA.

${ }^{6}$ Princeton University Observatory, Peyton Hall, Princeton, NJ 08544, USA.

${ }^{7}$ Department of Physics, Toho University, Funabashi, Chiba 274-8510, Japan.

${ }^{8}$ Institute of Astronomy, School of Science, University of Tokyo, 2-21-1 Osawa, Mitaka, Tokyo 181-0015, Japan.

${ }^{9}$ Optical and Infrared Astronomy Division, National Astronomical Observatory of Japan, 2-21-1 Osawa, Mitaka, Tokyo 181-8588, Japan.

${ }^{10}$ Department of Astronomy, Graduate School of Science, University of Tokyo 7-3-1, Hongo Bunkyo-ku, Tokyo 113-0033, Japan.

${ }^{11}$ Institute for Cosmic Ray Research, University of Tokyo, Kashiwa, 277-8582, Japan.

${ }^{12}$ Department of Astronomy, The Ohio State University, Columbus, OH 43210, USA.

${ }^{13}$ Department of Physics, Drexel University, 3141 Chestnut Street, Philadelphia, PA 19104, USA.

${ }^{14}$ Department of Astronomy and Astrophysics, The Pennsylvania State University, 525 Davey Laboratory, University Park, PA 16802, USA.

${ }^{15}$ Institute for Gravitation and the Cosmos,The Pennsylvania State University, 525 Davey Laboratory, University Park, PA 16802, USA.

${ }^{16}$ Department of Astronomy and Astrophysics, The University of Chicago, 5640 South Ellis Avenue, Chicago, IL 60637, USA.

${ }^{17}$ Enrico Fermi Institute, The University of Chicago, 5640 South Ellis Avenue, Chicago, IL 60637, USA.

${ }^{18}$ Kavli Institute for Cosmological Physics, University of Chicago, Chicago, IL 60637, USA.

${ }^{19}$ Center for Particle Astrophysics, Fermi National Accelerator Laboratory, P.O. Box 500, Batavia, IL 60510, USA.

${ }^{20}$ Department of Physics and Astronomy, York University, 4700 Keele Street, Toronto, Ontario, M3J 1P3, Canada.

${ }^{21}$ Space Telescope Science Institute, 3700 San Martin Drive, Baltimore, MD 21218, USA.
} 
We present the final statistical sample of lensed quasars from the Sloan Digital Sky Survey (SDSS) Quasar Lens Search (SQLS). The well-defined statistical lens sample consists of 26 lensed quasars brighter than $i=19.1$ and in the redshift range of $0.6<z<2.2$ selected from 50,836 spectroscopically confirmed quasars in the SDSS Data Release 7 (DR7), where we restrict the image separation range to $1^{\prime \prime}<\theta<20^{\prime \prime}$ and the $i$-band magnitude differences in two image lenses to be smaller than $1.25 \mathrm{mag}$. The SDSS DR7 quasar catalog also contains 36 additional lenses identified with various techniques. In addition to these lensed quasars, we have identified 81 pairs of quasars from follow-up spectroscopy, 26 of which are physically associated binary quasars. The statistical lens sample covers a wide range of image separations, redshifts, and magnitudes, and therefore is suitable for systematic studies of cosmological parameters and surveys of the structure and evolution of galaxies and quasars.

Subject headings: gravitational lensing: strong — quasars: general - cosmology: observations

\section{INTRODUCTION}

The Sloan Digital Sky Survey Quasar Lens Search (SQLS; Oguri et al. 2006, 2008a; Inada et al. 2008a, 2010) is a large survey for gravitationally lensed quasars among the spectroscopically confirmed quasars in the Sloan Digital Sky Survey (SDSS; York et al. 2000). The SQLS is designed for statistical analyses, and hence pays particular attention to the selection function of the lens sample by taking advantage of the homogeneous SDSS data set. For rigorous statistical analyses, the so-called "statistical sample" is designed to be complete within its prescribed limits. Well-defined statistical lens samples can be used for a number of cosmological and astrophysical applications. For instance, the number counts of strong lenses in a given source quasar population is a useful constraint on the cosmological constant (Turner 1990; Fukugita et al. 1990). In addition, one can study the evolution of massive galaxies using the statistics of strong lenses, including the redshift distribution of lens galaxies (Kochanek 1992; Ofek et al. 2003; Rusin et al. 2003; Rusin \& Kochanek 2005; Koopmans et al. 2006, 2009; Auger et al. 2010; Faure et al. 2011).

Statistical lens samples have been constructed from both radio and optical quasar surveys. For instance, the Hubble Space Telescope (HST) Snapshot survey (Maoz et al. 1993) includes five lenses discovered among 502 bright high-redshift quasars. The Cosmic-Lens All Sky Survey (CLASS; Myers et al. 2003; Browne et al. 2003) was the largest quasar lens survey before the SQLS, and contains 13 lenses identified from a sample of 8958 flat spec- 
trum radio sources for the statistically well-defined sample. However, a large source of the systematic error in the statistical analysis of the CLASS lens sample comes from the poorly characterized redshift distribution of the sources (e.g., Muñoz et al. 2003).

In addition to strongly lensed quasars, strongly lensed galaxies are also searched for. In particular, recent wide-field urveys have produced large samples of galaxy-galaxy lenses (e.g., Bolton et al. 2008a; Faure et al. 2008; Marshall et al. 2009; Féron et al. 2009; Kubo et al. 2010; Treu et al. 2011; More et al. 2012; Brownstein et al. 2012), which are principally useful for studies of the lens galaxies. Quasar lenses are more useful as cosmological probes because they represent a better understood source population and it is easier to characterize the selection effects of point sources. Moreover, time delays between multiple images of quasars provide additional information on cosmological parameters and the structure of the lens galaxies (e.g., Kochanek 2002; Saha et al. 2006; Oguri 2007; Keeton \& Moustakas 2009; Suyu et al. 2010; Paraficz \& Hiorth 2010; Courbin et al. 2011).

In Inada et al. (2010), we presented the SQLS statistical lens sample of 19 lenses from the full SDSS-I data set (DR5; Adelman-McCarthy et al. 2007). In this paper, we present the final result of the SQLS from SDSS Data Release 7 (DR7; Abazajian et al. 2009). DR7 is the final data release of SDSS-II, and consists of about $8000 \mathrm{deg}^{2}$ of spectroscopic coverage. We select lens candidates from the DR7 spectroscopic quasar catalog of 105,783 quasars from Schneider et al. (2010). The selection process is the same as that used for the DR3 and DR5 statistical samples (Inada et al. 2008a, 2010). As in previous SQLS papers, we present the results of confirming observations for all the candidates, including quasars that were shown not to be gravitational lenses. Several of the candidates are found to be binary quasars (physical pairs of quasars with small separations).

In this paper, we describe our source quasar sample in Section 2, and identify lens candidates in Section 3. Follow-up observations of the lens candidates are presented in Section 4 . Section 5 discusses the lens sample, and we summarize our results in Section 6. When necessary, we assume a flat universe with matter density $\Omega_{M}=0.275$, cosmological constant $\Omega_{\Lambda}=0.725$, and the Hubble constant $h=H_{0} /\left(100 \mathrm{~km} \mathrm{~s}^{-1} \mathrm{Mpc}^{-1}\right)=0.702$ (Komatsu et al. 2011).

\section{SOURCE QUASARS}

The SDSS consists of imaging and spectroscopic surveys of a quarter of the sky using a dedicated wide-field 2.5-meter telescope (Gunn et al. 2006) at the Apache Point Observatory in New Mexico, USA. The imaging survey uses five broad-band filters (ugriz, Fukugita et al. 
1996; Gunn et al. 1998; Doi et al. 2010). The automated pipeline leads to astrometric accuracy better than about 0".1 (Pier et al. 2003), and photometric zeropoint accuracies of about 0.01 magnitude (Hogg et al. 2001; Smith et al. 2002; Ivezić et al. 2004; Tucker et al. 2006; Padmanabhan et al. 2008). The spectroscopic targets selected based on the imaging data, including candidate quasars selected based on colors (Richards et al. 2002), are observed with multi-object fiber spectrographs covering $3800 \AA$ to $9200 \AA$ with a resolution of $R \sim 1800$ (Stoughton et al. 2002; Blanton et al. 2003). All the SDSS-I and SDSS-II data are publicly available (Stoughton et al. 2002; Abazajian et al. 2003, 2004, 2005, 2009; Adelman-McCarthy et al. 2006, 2007, 2008).

The source quasar sample is constructed following the procedures detailed in Oguri et al. (2006, hereafter Paper I), Inada et al. (2008a, hereafter Paper II), and Inada et al. (2010, hereafter Paper IV). An important difference from the DR5 study is that the DR7 quasar catalog contains quasars found in a series of "Special Plates" (see Adelman-McCarthy et al. 2006; Schneider et al. 2010). Most of the Special Plates were obtained in observations along the celestial equator at the Southern Galactic Cap, and used different selection algorithms from the main survey to explore the boundaries of the selection algorithms. The remainder comes from Sloan Extension for the Galactic Understanding and Exploration (SEGUE; Yanny et al. 2009). Clearly these quasars are not appropriate for our statistical lens sample, because they have quite different selection functions and redshift and magnitude distributions from the main survey. We do not include quasars from the Special Plates in the statistical lens sample.

For the statistical sample, we start with the 105,783 quasars in the DR7 spectroscopic survey selected over $8000 \mathrm{deg}^{2}$ (Schneider et al. 2010). As discussed above, we first remove 9,742 quasars in the Special Plates, as they are not suited for the statistical analysis. We then restrict the redshift range to $0.6<z<2.2$ and the $i$-band Point Spread Function (PSF) magnitude to $i<19.1$ after correcting for Galactic extinction based on Schlegel et al. (1998). The quasar target selection is almost complete over these redshift and magnitude ranges (see Richards et al. 2006; Paper I). In addition, we exclude quasars in poor seeing fields (PSF_WIDTH $>1$ 1". in $i$-band) where selection of small-separation lenses becomes very inefficient. These selection criteria lead to a sample of 50,836 source quasars. We give the catalog of the source quasars in Table 1.

\section{LENS CANDIDATES}

The SQLS uses two different algorithms for selecting lens candidates. "Morphological selection" is used to find smaller separation $(\theta \lesssim 2$."5) lensed quasars which are not deblended 
into multiple components by the SDSS photometric pipeline. The other is "color selection" to find large separation lenses $(\theta \gtrsim 2.5)$ whose lensed quasar components are found as separate objects in the SDSS image catalog.

The specific procedures of the algorithms and the selection functions are detailed in Papers I and II. In brief, morphological selection identifies quasars that are extended and poorly fit by the PSF. In morphological selection we check the parameter star_L, a logarithmic likelihood that the object is fit by the PSF, and the parameter objc_type, which assigns a classification of the object as star or galaxy. The algorithm was tested against many simulated lensed quasars in Paper I. We then fit the SDSS image of each system with a model consisting of two stellar components using GALFIT (Peng et al. 2002, 2010), and exclude objects that have large flux differences or have separations between the two PSF components that are too small in both the $u$ - and $i$-band SDSS images. This procedure is necessary to reject obvious single quasars and quasar-galaxy superpositions. We then visually inspect the images of candidates that survive the GALFIT selection step to exclude other obvious superpositions of a quasar and a galaxy. Of the 50,836 DR7 source quasars, 187 lens candidates are selected by the morphological selection criteria. Finally we compare the candidates with DR3 (Paper II) and DR5 (Paper IV) candidates, and find that 133 candidates have previously been examined. The remaining 54 morphological candidates are subject to detailed study in this paper.

We also identify lens candidates based on our color selection criteria for cases where the lensed images are deblended by the photometric pipeline. For each source quasar, we search for objects in the vicinity that have colors similar to those of the quasar. We select objects with an angular separation $\theta<20^{\prime \prime} 1$ and $i$-band magnitude differences $|\Delta i|<1$. 3 , in order to obtain a complete sample with image separations $1^{\prime \prime}<\theta<20^{\prime \prime}$ and $i$-band magnitude differences between two images $|\Delta i|<1.25$ with some allowance. We then compare optical and radio flux ratios, which are measured from the Faint Images of the Radio Sky at Twenty centimeters survey (FIRST; Becker et al. 1995), and exclude candidates that show clearly inconsistent flux ratios between the optical and radio. Given the resolution of the FIRST data, we examine the radio data only for candidates with image separations larger than $6^{\prime \prime}$. We also reject obvious quasar-galaxy pairs by visual inspection. Furthermore, we exclude low-redshift, large-separation pairs with no detectable lensing objects in the SDSS images, because at least one of the member galaxies of the putative lens group/cluster should be detectable for such lensing events (see Paper II for quantitative details). This leaves 340 color selected candidates out of 50,836 DR7 source quasars. Since 216 of these 340 candidates were previously examined in Paper II and Paper IV, we investigate the remaining 124 color candidates in this paper. 
To summarize, we identified 54 and 124 new candidates based on our morphological and color selection criteria, respectively, which required additional observations to complete the construction of the DR7 statistical lens sample. Two candidates are selected by both methods, because their image separations of $\theta \sim 2^{\prime \prime}-3^{\prime \prime}$ correspond to the transition of the image separation ranges probed by the two selection algorithms. Thus the total number of unique new candidates is 176 . Table 2 summarizes how many lens candidates are rejected at each step of the process.

\section{OBSERVATIONS}

\subsection{Summary of Follow-up Observations}

Among the 176 new lensed quasar candidates selected in the previous section, two systems are previously discovered lenses or binary quasars. One is SDSS J095122.57+263513.9 (FBQ0951+2635, Schechter et al. 1998), a gravitational lens with an image separation of $\theta=1^{\prime \prime}$.1 discovered in the FIRST Bright QSO Survey (Gregg et al. 1996). The other, SDSS J143002.66+071415.6, was shown to be a pair of quasars at $z=1.246$ and $z=1.261$ (Myers et al. 2008). It was discovered in the course of a binary quasar survey of the SDSS photometric quasar catalog of Richards et al. (2004).

We observed all the remaining 174 lens candidates using various telescope facilities, which are summarized in Tables 3 and 41 These observations consist of optical imaging, near-infrared imaging, and optical spectroscopy. We follow Paper II and Paper IV for the follow-up strategy, which is briefly summarized here. For the morphological candidates, we usually start with optical imaging under good seeing conditions (seeing FWHM $\lesssim 1^{\prime \prime}$ ) to see if the candidates have two or more stellar components and a lens galaxy between the stellar components. Most of the candidates are easily rejected by such observations, because they either have only a single component or show no sign of a lens galaxy. We carry out spectroscopy of multiple stellar components for candidates which show plausible lens galaxies in the follow-up images. We obtained spectra of 13 morphological candidates, 6 of which are true gravitational lenses. Two additional candidates, SDSS J125107.57+293540.5 and SDSS J133018.64+181032.1, were confirmed as gravitational lenses without needing spectroscopy because of their obvious quadruple image configurations. For the 122 colorselected candidates, excluding the two candidates that were also selected as morphological

\footnotetext{
${ }^{1}$ We provide a list of all the 520 targets, including those investigated in Paper II and Paper IV, and the summaries of observations of these candidates on the SQLS webpage http://www-utap.phys.s.u-tokyo.ac.jp/ ^sdss/sqls/.
} 
candidates, we discovered many pairs of quasars in the spectroscopic follow-up observations (see $\S 4.2$ and $\S$ 5.4). Based on the similarity of the spectra of the stellar components and the detection of the lensing object, we conclude that only 2 of the 122 color candidates are true gravitational lens systems. To summarize, these follow-up observations led to the discoveries of 10 gravitational lenses, which are reported in Kayo et al. (2007), Kavo et al. (2010), Oguri et al. (2008d), Jackson et al. (2012), Rusu et al. (2012, in preparation), and Inada et al. (2012, in preparation).

\subsection{Note on individual objects}

In addition to our confirmed gravitational lens systems, we found 24 objects that have multiple quasar components. Here we discuss the three binary quasar pairs with velocity differences smaller than $1000 \mathrm{~km} \mathrm{~s}^{-1}$ and a morphological candidate which is a potential gravitational lens outside the statistical sample.

SDSS J094234.97+231031.1: Our spectroscopy at the 6.5-meter MMT indicates that both stellar components, separated by $\theta=2^{\prime \prime} .5$, have similar spectra of a quasar at $z=1.833$, but the shapes of the C IV and C III] emission lines are significantly different. We also find no evidence for a lens between them in images taken with UH8k at the University of Hawaii 2.2meter telescope ( $R$ and $I$-band) or with NICFPS on the Apache Point Observatory 3.5-meter telescope $(K$-band). Therefore, we conclude that this object is a binary quasar.

SDSS J112235.03+232634.9: This is a small separation $\left(\theta \sim 0^{\prime \prime} 7\right)$ lens candidate identified based on its morphology. We obtained spectra with FOCAS (Kashikawa et al. 2002) at the Subaru 8.2-meter telescope and I-band images with the University of Hawaii 2.2-meter telescope, but these observations have been inconclusive. Because of the small image separation, this system would not be included in the statistical lens sample even if confirmed.

SDSS J124257.32+254303.0: Spectra taken at the Subaru 8.2-meter telescope indicate that the two stellar components separated by $\theta=2$ ". 8 are quasars at slightly different redshifts of $z=0.827$ and $z=0.824\left(\Delta v \sim 560 \mathrm{~km} \mathrm{~s}^{-1}\right)$, as measured from the $\mathrm{Mg}$ II emission lines. Images taken with tek2k at the University of Hawaii 2.2-meter telescope ( $I$-band) show extended emission between the two components, but we interpret this as host galaxy emission of one or both quasars, particularly given the low quasar redshifts.

SDSS J143350.94+145008.1: While the two stellar components with a separation of $\theta=3 . .3$ have similar redshifts of $z=1.506$, based on spectroscopy at the Apache Point Observatory 3.5-meter telescope, the two spectra are markedly different. One component 
has broad absorption features on the blue side of the C IV and C III] emission lines, which are not seen in the spectrum of the other component. In addition, imaging at the University of Hawaii 2.2-meter telescope ( $I$-band) shows no sign of a lens galaxy. We conclude that this object is a binary quasar.

\section{LENSED QUASAR CATALOG}

\subsection{Statistical Sample}

We define the statistical lens sample to include objects with an image separation in the range of $1^{\prime \prime}<\theta<20^{\prime \prime}$ and an $i$-band magnitude difference less than 1.25 mag for doubles, as was adopted in Paper II and Paper IV. The ranges are determined such that the lens candidate selection is almost complete (see Paper I). Among the 11 new lensed quasars since DR5 selected by these algorithms, 7 lenses satisfy the criteria. Together with 19 lenses from the DR5 sample (Paper IV), the statistical lens sample consists of 26 lenses selected from a sample of 50,836 source quasars.

The properties of this statistical lens sample are summarized in Table 5. Among the 26 lenses there are only four four-image lenses (SDSS J092455.79+021924.9, PG1115+080, SDSS J125107.57+293540.5, and SDSS J133018.64+181032.1) and one five-image lens, the cluster lens SDSS J100434.92+411242.7 (Inada et al. 2003b; Oguri et al. 2004b; Inada et al. 2005a). In addition to basic parameters such as source redshifts and $i$-band magnitudes, we show the magnitudes of the lens galaxies measured in the discovery papers or by the HST from the CASTLES webpage if available. We also revisited the lens redshifts for some of the lens systems. New spectroscopy with Gemini/GMOS allows us to easily measure the lens redshift of SDSS J100128.61+502756.8 to be $z=0.415$ (N. Inada et al., in preparation). A re-examination of the spectrum of SDSS J105545.45+462839.4 (Kayo et al. 2010) shows Mg and $\mathrm{Na}$ absorption lines in the spectrum of the lens galaxy, from which we determine the lens redshift to be $z=0.388$. We detected $\mathrm{Mg}$ II absorption lines in the quasar spectrum of SDSS J102111.01+491330.3 at a redshift of $z=0.451$ that agrees well with the lens redshift expected from the color and brightness of the lens galaxy.

\subsection{Additional Lensed Quasars}

As in previous SQLS data release papers (Paper II and Paper IV), we have identified

several new lenses from the DR7 quasar catalog that are not included in the statistical lens sample. Table 6 summarizes these 36 additional lenses, 18 of which are new quasars in 
the DR7 catalog that were not include in the DR5 quasar catalog. Many were discovered by the SQLS simply by applying our selection algorithms to quasars that are outside the redshift, magnitude, and image separation ranges of the statistical lens sample (see also Paper IV). The large-separation lens SDSS J102913.94+262317.9 (Inada et al. 2006b; Oguri et al. 2008c) is included in this category because its image separation of $\theta=22.5$ is slightly larger than the image separation limit for the statistical lens sample. Several lenses were also discovered by examining the morphology of SDSS quasars in the UKIDSS (UKIRT Infrared Deep Sky Survey; Lawrence et al. 2007), by taking advantage of the higher image quality and the more dominant contribution of lens galaxies in the near-infrared (Jackson et al. 2008, 2009, 2012).

We can also update two of these lens redshifts. The lens redshift of SDSS J125819.24+165717.6 measured in our follow-up Gemini/GMOS spectroscopy is $z=0.505$ (N. Inada et al., in preparation). From a re-examination of the spectrum of SDSS J130451.52+161138.2 (Kayo et al. 2010), we conclude that the lens redshift is likely to be $z=0.373$.

\subsection{Properties of the Lens Samples}

In Figure 1, we show the distributions of our lens samples in several variables. The image separation distribution clearly indicates that our sample covers a wide range of lens types, ranging from lensing by single galaxies $\left(\theta \sim 1^{\prime \prime}\right)$ to clusters of galaxies $\left(\theta \gtrsim 10^{\prime \prime}\right)$. The distribution is in reasonable agreement with that of strongly lensed galaxies in the CFHT

Legacy Survey (More et al. 2012) as well as with theoretical expectations (e.g., Oguri 2006). Our lens sample contains many lensed quasars with $i \sim 19$, close to the magnitude limit of SDSS spectroscopic quasars in this redshift range. The average source redshift of the statistical sample is $\left\langle z_{s}\right\rangle=1.56$, whereas that of the entire sample is $\left\langle z_{s}\right\rangle=1.95$. The lens redshifts are also distributed over a wide range, from $z_{l} \sim 0.2$ to $z_{l} \sim 1$.

The image separations are expected to be larger for more massive lensing objects, but with a sharp cutoff between galaxy and cluster mass scales due to the effect of baryon cooling (e.g., Kochanek \& White 2001). For the singular isothermal sphere model, the image separation is $\theta=8 \pi\left(D_{l s} / D_{o s}\right)\left(\sigma_{v} / c\right)^{2}$, where $D_{l s}$ and $D_{o s}$ are the angular diameter distances between the lens and the source and between the observer and the source, respectively. The well-known scaling relation between velocity dispersions $\sigma_{v}$ and galaxy luminosities (Faber \& Jackson 1976) suggests that the image separations should correlate with galaxy luminosities. The correlation between the image separation $\theta$ and the $r$-band absolute magnitude of the lens galaxy is plotted in Figure 2, The absolute magnitudes are computed from apparent magnitudes of lens galaxies listed in Tables 5 and 6 using the cross-filter 
$K$-correction from a template spectrum of an elliptical galaxy by Coleman et al. (1980). We see a clear correlation between $\theta$ and $M_{r}$, such that lenses with larger image separations have more luminous lens galaxies, consistent with the expectation and earlier lensing results (e.g., Rusin et al. 2003; Bolton et al. 2008b). Independently of the mass model, the image separation scales with the distance ratio $D_{l s} / D_{o s}$, and thus we also consider the scaled image separation $\theta\left(D_{l s} / D_{o s}\right)^{-1}$ to remove the dependence on a distance. As expected, the scaled image separations show a tighter correlation with the absolute magnitudes. A few lenses with $\theta \gtrsim 10^{\prime \prime}$ are offset from the correlation. These are cluster lenses where there is a much larger contribution of dark matter inside the Einstein radius (e.g., Kochanek \& White 2001). We note that two previously known subarcsecond lenses PMN J0134-0931 and FBQ0951+2635 show large deviations from the correlation. One of the reasons is that their lens galaxies are likely to be spiral galaxies for which the Faber-Jackson relation cannot be applied, but another reason might be due to errors in magnitudes or redshifts estimates for their lens galaxies.

The distance-scaled image separation $\theta\left(D_{l s} / D_{o s}\right)^{-1}$ should be independent of redshift for the standard singular isothermal sphere model. Hence by adopting the Faber-Jackson relation (Faber \& Jackson 1976), the correlation between the velocity dispersion $\sigma_{v}$ and the absolute magnitude of early-type galaxies, we can predict the expected $\theta\left(D_{l s} / D_{o s}\right)^{-1}$ $M_{r}$ correlation. Note that most of lens galaxies in our sample are early-type galaxies. In Figure 2 we also show the correlation expected from the Faber-Jackson relation observed in the SDSS data (Bernardi et al. 2003). Here we include luminosity evolution by extrapolating the redshift evolution measured by Bernardi et al. (2003) to $z=0.5$, which is roughly the median lens redshift for our sample (see Figure 1).

We find that the predicted relation properly reproduces the observed slope, except for the largest separation lenses, where the lens galaxies are much fainter than the relation predicts due to the dominant contribution of dark matter to these image separations. On the other hand, the normalization is slightly offset, in the sense that the observed lens galaxies are on average fainter than the relation predicts. One cause of this shift is a bias created by the large scatter of the Faber-Jackson relation (Kochanek 1994). Bernardi et al. (2003) found a scatter in $\log \sigma_{v}$ of $\sim 0.07$ at fixed galaxy luminosity. Since the lensing cross section is proportional to $\sigma_{v}^{4}$, the lensing-weighted average of the velocity dispersion is given by the average over the Gaussian in $\log \sigma_{v}$ times $\sigma_{v}^{4}$. Thus the lensing bias shifts the mean $\log \sigma_{v}$ by $\Delta\left(\log \sigma_{v}\right) \sim 0.05$, which roughly corresponds to the image separation shift of $\Delta(\log \theta)=2 \Delta\left(\log \sigma_{v}\right) \sim 0.1$. Including this lensing bias in the predicted correlation agrees much better with observations, as shown in Figure 2, This comparison highlights the importance of understanding the selection effects for studying galaxy properties from observed strong lens systems. 


\subsection{Binary Quasars and Projected Quasar Pairs}

One important by-product of the SQLS is the discovery of many quasar pairs and binary quasars. In total we have identified 81 pairs of quasars, 26 of which are quasar pairs with velocity differences smaller than the $2000 \mathrm{~km} \mathrm{~s}^{-1}$ threshold used for the statistical analysis of binary quasars in Hennawi et al. (2006a). Most of these binary quasars and projected quasar pairs are new. In particular, we have identified 12 pairs of quasars with angular separations smaller than $3^{\prime \prime}$. Detailed explorations of these small separation binary quasars are important for understanding the possible connection of quasar activity with galaxy mergers (e.g., Green et al. 2010). These quasar pairs also provide useful information about the nature of quasars by constraining their small-scale correlation function (Hennawi et al. 2006a; Mvers et al. 2008) and the distribution of absorbers around quasars (Bowen et al. 2006; Hennawi et al. 2006b; Tytler et al. 2009).

\section{SUMMARY}

We have presented the final statistical lens sample of the SQLS from the SDSS DR7 quasar catalog (Schneider et al. 2010). We observed 176 lensed quasar candidates selected from 50,836 quasars with various facilities to determine whether they are in fact lenses. Together with the results we reported in Paper II and Paper IV, we have constructed a sample of 26 lensed quasars within a well-specified separation range and magnitude difference. The well-defined statistical sample presented in the paper will be essential for studying dark energy as well as the evolution of massive clusters. We present a detailed statistical analysis of this final lens sample in a separate paper (Oguri et al. 2012, paper VI). In addition to the statistical sample, the DR7 quasar catalog contains at least 36 additional lenses identified with various techniques. These additional lenses will also be useful for detailed future studies, including precise mass modeling from high-resolution astrometry, time delay measurements, the measurement of the lens velocity dispersion, and the study of high-redshift quasars through host galaxy or microlensing observations. The SQLS has also discovered many small-separation binary quasars and projected quasar pairs, which may provide a clue to the origin and nature of quasar activity.

The largest quasar lens survey before SQLS was CLASS, which contains 13 lenses in their statistical sample selected from 8958 flat spectrum radio sources, plus 9 additional lenses (Myers et al. 2003; Browne et al. 2003) Thus, the final SQLS sample more than doubles the number of quasar lenses in a single statistical lens sample, which is a significant improvement. Furthermore, an advantage of SQLS over CLASS is that the redshifts of all the source quasars are known, which allows us to conduct more robust statistical analyses. 
It is expected that future wide-field optical imaging surveys such as Pan-STARRS, Hyper Suprime-cam, the Dark Energy Survey, and the Large Synoptic Survey Telescope will find many more strongly lensed quasars (Oguri \& Marshall 2010). These future surveys plan to explore the time domain, which can make use of the time variability of quasar images to locate lensed quasars efficiently (Kochanek et al. 2006; Lacki et al. 2009). In addition, the ongoing SDSS-III survey (Aihara et al. 2011; Eisenstein et al. 2011) plans to take spectra of more than 100,000 quasars at $2.2<z<3$ selected from the SDSS imaging data (Ross et al. 2012). We plan to apply the same algorithms used in the SQLS to the SDSS-III data to discover many more lensed quasars.

Use of the UH 2.2-m telescope and the UKIRT 3.8-m telescope for the observations is supported by the National Astronomical Observatory of Japan (NAOJ). Based in part on observations obtained with the Apache Point Observatory 3.5-meter telescope, which is owned and operated by the Astrophysical Research Consortium. Based in part on data collected at Subaru Telescope, which is operated by the National Astronomical Observatory of Japan, and obtained from the SMOKA, which is operated by the Astronomy Data Center, National Astronomical Observatory of Japan. Based in part on observations obtained at the Gemini Observatory, which is operated by the Association of Universities for Research in Astronomy, Inc., under a cooperative agreement with the NSF on behalf of the Gemini partnership: the National Science Foundation (United States), the Science and Technology Facilities Council (United Kingdom), the National Research Council (Canada), CONICYT (Chile), the Australian Research Council (Australia), Ministério da Ciência e Tecnologia (Brazil) and Ministerio de Ciencia, Tecnología e Innovación Productiva (Argentina). Telescopio Nazionale Galileo (TNG) operated on the island of La Palma by the Fundacion Galileo Galilei of the INAF (Istituto Nazionale di Astrofisica) at the Spanish Observatorio del Roque de los Muchachos of the Instituto de Astrofisica de Canarias.

This work was supported in part by the FIRST program "Subaru Measurements of Images and Redshifts (SuMIRe)", World Premier International Research Center Initiative (WPI Initiative), MEXT, Japan, and Grant-in-Aid for Scientific Research from the JSPS (23740161). This work is supported in part by JSPS Core-to-Core Program "International Research Network for Dark Energy". N. I. acknowledges support from MEXT KAKENHI 21740151. M. A. S. acknowledges the support of NSF grant AST-0707266. C. E. R. acknowledges the support of the JSPS Research Fellowship. C. S. K. is supported by NSF grant AST-1009756. The Institute for Gravitation and the Cosmos is supported by the Eberly College of Science and the Office of the Senior Vice President for Research at the Pennsylvania State University.

Funding for the SDSS and SDSS-II has been provided by the Alfred P. Sloan Foundation, 
the Participating Institutions, the National Science Foundation, the U.S. Department of Energy, the National Aeronautics and Space Administration, the Japanese Monbukagakusho, the Max Planck Society, and the Higher Education Funding Council for England. The SDSS Web Site is http://www.sdss.org/,

The SDSS is managed by the Astrophysical Research Consortium for the Participating Institutions. The Participating Institutions are the American Museum of Natural History, Astrophysical Institute Potsdam, University of Basel, Cambridge University, Case Western Reserve University, University of Chicago, Drexel University, Fermilab, the Institute for Advanced Study, the Japan Participation Group, Johns Hopkins University, the Joint Institute for Nuclear Astrophysics, the Kavli Institute for Particle Astrophysics and Cosmology, the Korean Scientist Group, the Chinese Academy of Sciences (LAMOST), Los Alamos National Laboratory, the Max-Planck-Institute for Astronomy (MPIA), the Max-Planck-Institute for Astrophysics (MPA), New Mexico State University, Ohio State University, University of Pittsburgh, University of Portsmouth, Princeton University, the United States Naval Observatory, and the University of Washington.

\section{REFERENCES}

Abazajian, K., et al. 2003, AJ, 126, 2081

Abazajian, K., et al. 2004, AJ, 128, 502

Abazajian, K., et al. 2005, AJ, 129, 1755

Abazajian, K. N., et al. 2009, ApJS, 182, 543

Adelman-McCarthy, J. K., et al. 2006, ApJS, 162, 38

Adelman-McCarthy, J. K., et al. 2007, ApJS, 172, 634

Adelman-McCarthy, J. K., et al. 2008, ApJS, 175, 297

Aihara, H., et al. 2011, ApJS, 193, 29

Auger, M. W., et al. 2010, ApJ, 724, 511

Bade, N., Siebert, J., Lopez, S., Voges, W., \& Reimers, D. 1997, A\&A, 317, L13

Becker, R. H., White, R. L., \& Helfand, D. J. 1995, ApJ, 450, 559

Bernardi, M., et al. 2003, AJ, 125, 1849 
Blanton, M. R., Lin, H., Lupton, R. H., Maley, F. M., Young, N., Zehavi, I., \& Loveday, J. 2003, AJ, 125, 2276

Bolton, A. S., et al. 2008a, ApJ, 682, 964

Bolton, A. S., et al. 2008b, ApJ, 684, 248

Bowen, D. V., et al. 2006, ApJ, 645, L105

Browne, I. W. A., et al. 2003, MNRAS, 341, 13

Brownstein, J. R., et al. 2012, ApJ, 744, 41

Coleman, G. D., Wu, C.-C., \& Weedman, D. W. 1980, ApJS, 43, 393

Courbin, F., et al. 2011, A\&A, 536, A53

Doi, M., et al. 2010, AJ, 139, 1628

Eigenbrod, A., Courbin, F., Dye, S., Meylan, G., Sluse, D., Vuissoz, C., \& Magain, P. 2006a, A\&A, 451, 747

Eigenbrod, A., Courbin, F., Meylan, G., Vuissoz, C., \& Magain, P. 2006b, A\&A, 451, 759

Eigenbrod, A., Courbin, F., \& Meylan, G. 2007, A\&A, 465, 51

Eisenstein, D. J., et al. 2011, AJ, 142, 72

Faber, S. M., \& Jackson, R. E. 1976, ApJ, 204, 668

Faure, C., et al. 2008, ApJS, 176, 19

Faure, C., et al. 2011, A\&A, 529, A72

Féron, C., Hjorth, J., McKean, J. P., \& Samsing, J. 2009, ApJ, 696, 1319

Fukugita, M., Futamase, T., \& Kasai, M. 1990, MNRAS, 246, 24

Fukugita, M., Ichikawa, T., Gunn, J. E., Doi, M., Shimasaku, K., \& Schneider, D. P. 1996, AJ, 111, 1748

Goicoechea, L. J., \& Shalyapin, V. N. 2010, ApJ, 708, 995

Green, P. J., et al. 2010, ApJ, 710, 1578

Gregg, M. D., et al. 1996, AJ, 112, 407 
Gregg, M. D., Lacy, M., White, R. L., Glikman, E., Helfand, D., Becker, R. H., \& Brotherton, M. S. 2002, ApJ, 564, 133

Gunn, J. E., et al. 1998, AJ, 116, 3040

Gunn, J. E., et al. 2006, AJ, 131, 2332

Hagen, H.-J., \& Reimers, D. 2000, A\&A, 357, L29

Hall, P. B., Richards, G. T., York, D. G., Keeton, C. R., Bowen, D. V., Schneider, D. P., Schlegel, D. J., \& Brinkmann, J. 2002, ApJ, 575, L51

Hennawi, J. F., et al. 2006a, AJ, 131, 1

Hennawi, J. F., et al. 2006b, ApJ, 651, 61

Hogg, D. W., Finkbeiner, D. P., Schlegel, D. J., \& Gunn, J. E. 2001, AJ, 122, 2129

Inada, N., et al. 2003a, AJ, 126, 666

Inada, N., et al. 2003b, Nature, 426, 810

Inada, N., et al. 2005a, PASJ, 57, L7

Inada, N., et al. 2005b, AJ, 130, 1967

Inada, N., et al. 2006a, AJ, 131, 1934

Inada, N., et al. 2006b, ApJ, 653, L97

Inada, N., et al. 2007, AJ, 133, 206

Inada, N., et al. 2008a, AJ, 135, 496 (Paper II)

Inada, N., Oguri, M., Falco, E. E., Broadhurst, T. J., Ofek, E. O., Kochanek, C. S., Sharon, K., \& Smith, G. P. 2008b, PASJ, 60, L27

Inada, N., et al. 2009, AJ, 137, 4118

Inada, N., et al. 2010, AJ, 140, 403 (Paper IV)

Ivezić, Ž., et al. 2004, Astronomische Nachrichten, 325, 583

Jackson, N., Ofek, E. O., \& Oguri, M. 2008, MNRAS, 387, 741

Jackson, N., Ofek, E. O., \& Oguri, M. 2009, MNRAS, 398, 1423 
Jackson, N., Rampadarath, H., Ofek, E. O., Oguri, M., \& Shin, M.-S. 2012, MNRAS, 419, 2014

Johnston, D. E., et al. 2003, AJ, 126, 2281

Kashikawa, N., et al. 2002, PASJ, 54, 819

Kayo, I., et al. 2007, AJ, 134, 1515

Kayo, I., Inada, N., Oguri, M., Morokuma, T., Hall, P. B., Kochanek, C. S., \& Schneider, D. P. 2010, AJ, 139, 1614

Keeton, C. R., \& Moustakas, L. A. 2009, ApJ, 699, 1720

Kneib, J.-P., Cohen, J. G., \& Hjorth, J. 2000, ApJ, 544, L35

Kochanek, C. S. 1992, ApJ, 384, 1

Kochanek, C. S. 1994, ApJ, 436, 56

Kochanek, C. S. 2002, ApJ, 578, 25

Kochanek, C. S., \& White, M. 2001, ApJ, 559, 531

Kochanek, C. S., Mochejska, B., Morgan, N. D., \& Stanek, K. Z. 2006, ApJ, 637, L73

Komatsu, E., et al. 2011, ApJS, 192, 18

Koopmans, L. V. E., Treu, T., Bolton, A. S., Burles, S., \& Moustakas, L. A. 2006, ApJ, 649, 599

Koopmans, L. V. E., et al. 2009, ApJ, 703, L51

Kubo, J. M., 2010, ApJ, 724, L137

Kundic, T., Cohen, J. G., Blandford, R. D., \& Lubin, L. M. 1997, AJ, 114, 507

Lacki, B. C., Kochanek, C. S., Stanek, K. Z., Inada, N., \& Oguri, M. 2009, ApJ, 698, 428

Lacy, M., Gregg, M., Becker, R. H., White, R. L., Glikman, E., Helfand, D., \& Winn, J. N. 2002, AJ, 123, 2925

Lawrence, A., et al. 2007, MNRAS, 379, 1599

Lubin, L. M., Fassnacht, C. D., Readhead, A. C. S., Blandford, R. D., \& Kundić, T. 2000, AJ, 119, 451 
Magain, P., Surdej, J., Swings, J.-P., Borgeest, U., \& Kayser, R. 1988, Nature, 334, 325

Maoz, D., et al. 1993, ApJ, 409, 28

Marshall, P. J., Hogg, D. W., Moustakas, L. A., Fassnacht, C. D., Bradač, M., Schrabback, T., \& Blandford, R. D. 2009, ApJ, 694, 924

McGreer, I. D., et al. 2010, AJ, 140, 370

More, A., et al. 2012, ApJ, in press (arXiv:1109.1821)

Morgan, N. D., Becker, R. H., Gregg, M. D., Schechter, P. L., \& White, R. L. 2001, AJ, 121, 611

Morgan, N. D., Snyder, J. A., \& Reens, L. H. 2003, AJ, 126, 2145

Morokuma, T., et al. 2007, AJ, 133, 214

Muñoz, J. A., Falco, E. E., Kochanek, C. S., McLeod, B. A., \& Mediavilla, E. 2003, ApJ, 605,614

Myers, S. T., et al. 1999, AJ, 117, 2565

Myers, S. T., et al. 2003, MNRAS, 341, 1

Myers, A. D., et al. 2007, ApJ, 658, 99

Myers, A. D., Richards, G. T., Brunner, R. J., Schneider, D. P., Strand, N. E., Hall, P. B., Blomquist, J. A., \& York, D. G. 2008, ApJ, 678, 635

Ofek, E. O., Rix, H.-W., \& Maoz, D. 2003, MNRAS, 343, 639

Ofek, E. O., Oguri, M., Jackson, N., Inada, N., \& Kayo, I. 2007, MNRAS, 382, 412

Oguri, M. 2006, MNRAS, 367, 1241

Oguri, M. 2007, ApJ, 660, 1

Oguri, M., \& Marshall, P. J. 2010, MNRAS, 405, 2579

Oguri, M., et al. 2004a, PASJ, 56, 399

Oguri, M., et al. 2004b, ApJ, 605, 78

Oguri, M., et al. 2005, ApJ, 622, 106 
Oguri, M., et al. 2006, AJ, 132, 999 (Paper I)

Oguri, M., et al. 2008a, AJ, 135, 512 (Paper III)

Oguri, M., et al. 2008b, AJ, 135, 520

Oguri, M., et al. 2008c, ApJ, 676, L1

Oguri, M., Inada, N., Blackburne, J. A., Shin, M.-S., Kayo, I., Strauss, M. A., Schneider, D. P., \& York, D. G. 2008d, MNRAS, 391, 1973

Oguri, M., et al. 2012, AJ, in press, arXiv:1203.1088 (Paper VI)

Oscoz, A., Serra-Ricart, M., Mediavilla, E., Buitrago, J., \& Goicoechea, L. J. 1997, ApJ, 491, L7

Padmanabhan, N., et al. 2008, ApJ, 674, 1217

Paraficz, D., \& Hjorth, J. 2010, ApJ, 712, 1378

Peng, C. Y., Ho, L. C., Impey, C. D., \& Rix, H.-W. 2002, AJ, 124, 266

Peng, C. Y., Ho, L. C., Impey, C. D., \& Rix, H.-W. 2010, AJ, 139, 2097

Pier, J. R., Munn, J. A., Hindsley, R. B., Hennessy, G. S., Kent, S. M., Lupton, R. H., \& Ivezić, Ž. 2003, AJ, 125, 1559

Pindor, B., et al. 2004, AJ, 127, 1318

Pindor, B., et al. 2006, AJ, 131, 41

Reimers, D., Hagen, H.-J., Baade, R., Lopez, S., \& Tytler, D. 2002, A\&A, 382, L26

Richards, G. T., et al. 2002, AJ, 123, 2945

Richards, G. T., et al. 2004, ApJS, 155, 257

Richards, G. T., et al. 2006, AJ, 131, 2766

Ross, N. P., et al. 2012, ApJS, 199, 3

Rusin, D., et al. 2003, ApJ, 587, 143

Rusin, D., \& Kochanek, C. S. 2005, ApJ, 623, 666

Rusu, C. E., et al. 2011, ApJ, 738, 30 
Saha, P., Coles, J., Macciò, A. V., \& Williams, L. L. R. 2006, ApJ, 650, L17

Schechter, P. L., Gregg, M. D., Becker, R. H., Helfand, D. J., \& White, R. L. 1998, AJ, 115, 1371

Schlegel, D. J., Finkbeiner, D. P., \& Davis, M. 1998, ApJ, 500, 525

Schneider, D. P., et al. 2010, AJ, 139, 2360

Sluse, D., Courbin, F., Eigenbrod, A., \& Meylan, G. 2008, A\&A, 492, L39

Smith, J. A., et al. 2002, AJ, 123, 2121

Stoughton, C., et al. 2002, AJ, 123, 485

Surdej, J., et al. 1987, Nature, 329, 695

Suyu, S. H., Marshall, P. J., Auger, M. W., Hilbert, S., Blandford, R. D., Koopmans, L. V. E., Fassnacht, C. D., \& Treu, T. 2010, ApJ, 711, 201

Treu, T., et al. 2011, MNRAS, 417, 1601

Tucker, D. L., et al. 2006, Astronomische Nachrichten, 327, 821

Turner, E. L. 1990, ApJ, 365, L43

Tytler, D., et al. 2009, MNRAS, 392, 1539

Walsh, D., Carswell, R. F., \& Weymann, R. J. 1979, Nature, 279, 381

Weymann, R. J., et al. 1980, Nature, 285, 641

Winn, J. N., Lovell, J. E. J., Chen, H.-W., Fletcher, A. B., Hewitt, J. N., Patnaik, A. R., \& Schechter, P. L. 2002, ApJ, 564, 143

Yanny, B., Rockosi, C., Newberg, H. J., et al. 2009, AJ, 137, 4377

York, D. G., et al. 2000, AJ, 120, 1579

Young, P., Gunn, J. E., Oke, J. B., Westphal, J. A., \& Kristian, J. 1980, ApJ, 241, 507 
Table 1. Source Quasars

\begin{tabular}{ccc}
\hline \hline Name & Redshift & $i$ \\
\hline SDSSJ000009.26+151754.5 & 1.1985 & 19.057 \\
SDSSJ000009.42-102751.9 & 1.8449 & 18.698 \\
SDSSJ000013.14+141034.6 & 0.9582 & 19.028 \\
SDSSJ000017.38-085123.7 & 1.2491 & 18.204 \\
SDSSJ000024.02+152005.4 & 0.9889 & 18.969 \\
\hline
\end{tabular}

Note. - The redshift and $i$-band magnitude (Galactic extinction corrected) distributions of the source quasars are presented in their entirety in the electronic edition of the Astronomical Journal. The first five rows are shown here for guidance regarding its form and content. See also the SQLS webpage (http://www-utap.phys.s.u-tokyo.ac.jp/ ^sdss/sqls/) for the list of the 50,836 quasars. 
Table 2. Number of Candidates at Each Stage of the Selection Process

\begin{tabular}{|c|c|}
\hline Candidate Selection Process & Number \\
\hline SDSS DR7 spectroscopically confirmed quasars & 105,783 \\
\hline Quasars in the special plates & $-9,742$ \\
\hline Quasars not passing the criteria $(0.6<z<2.2, i<19.1$, and PSF_WIDTH $<1$ ". 8$)$ & $-45,205$ \\
\hline Source quasars to complete the final statistical lens sample & $50,836^{\mathrm{a}}$ \\
\hline Initial morphologically selected candidates (using star_L in ugri) & 1,535 \\
\hline Rejected by GALFIT fitting & $-1,338$ \\
\hline Rejected by visual inspection & -10 \\
\hline Final morphologically selected candidates & 187 \\
\hline Studied in Paper II and Paper IV & -133 \\
\hline Morphologically selected candidates studied in this paper & 54 \\
\hline Initial color selected candidates & 527 \\
\hline Rejected by FIRST image check & -33 \\
\hline Rejected by visual inspection & -18 \\
\hline Rejected by searching for possible lens objects & -136 \\
\hline Final color selected candidates & 340 \\
\hline Studied in Paper II and Paper IV & -216 \\
\hline Color selected candidates studied in this paper & 124 \\
\hline Final total (morphological+color) candidates for follow-up & $520^{\mathrm{b}}$ \\
\hline Studied in Paper II and Paper IV & $-344^{\mathrm{b}}$ \\
\hline Final total candidates studied in this paper & $176^{\mathrm{b}}$ \\
\hline
\end{tabular}

${ }^{a}$ Among the 50,836 quasars, 35,623 quasars were checked in Paper IV. The number of source quasars in Paper IV is 36,287 , but 655 DR5 quasars are included in the special plates, or do not meet the criteria to make our source quasar sample in the DR7 quasar catalog. 
${ }^{\mathrm{b}}$ Seven candidates are selected by both the morphological and color selection algorithms, five of which were already studied in Paper II and Paper IV. The remaining two candidates selected by both the algorithms are listed in Table 3 . 
Table 3. DR7 Morphologically Selected Candidates

\begin{tabular}{|c|c|c|c|c|c|c|c|c|}
\hline Object & Redshift $^{\mathrm{a}}$ & $i^{\mathrm{b}}$ & $\theta_{\mathrm{SDSS}}^{\mathrm{c}}$ & $|\Delta i|^{\mathrm{c}}$ & Image $^{d}$ & $\operatorname{Spec}^{\mathrm{d}}$ & Comment & Ref. \\
\hline SDSS J074248.40+270412.2 & 0.626 & 19.05 & 0.78 & 1.74 & $\mathrm{Te}(\mathrm{I})$ & $\cdots$ & single QSO & $\cdots$ \\
\hline SDSS J081405.56+582840.9 & 1.429 & 18.24 & 0.50 & 0.94 & $\mathrm{Te}(\mathrm{I})$ & $\cdots$ & single QSO & $\cdots$ \\
\hline SDSS J090404.15+151254.5 & 1.825 & 17.50 & 1.04 & 1.64 & $\mathrm{Te}(\mathrm{V}, \mathrm{R}, \mathrm{I}), \mathrm{Op}(\mathrm{I})$ & $\mathrm{FO}$ & SDSS lens & 1 \\
\hline SDSS J090645.62+122512.8 & 0.602 & 19.09 & 0.63 & 0.99 & $\mathrm{Te}(\mathrm{I})$ & $\cdots$ & single QSO & $\cdots$ \\
\hline SDSS J092718.37+211357.4 & 1.845 & 17.24 & 2.36 & 0.21 & $\mathrm{Te}(\mathrm{I})$ & $\cdots$ & no lensing object & $\cdots$ \\
\hline SDSS J093139.97+402548.4 & 1.028 & 18.35 & 0.43 & 0.21 & $\mathrm{SP}(\mathrm{i})$ & $\cdots$ & single QSO & $\cdots$ \\
\hline SDSS J093527.90+232525.0 & 2.014 & 19.04 & 1.78 & 1.98 & $\mathrm{Te}(\mathrm{I})$ & $\ldots$ & $\mathrm{QSO}+$ galaxy & $\ldots$ \\
\hline SDSS J095036.63+261706.1 & 1.264 & 18.60 & 0.44 & 0.96 & $\mathrm{Te}(\mathrm{I})$ & $\cdots$ & single QSO & $\cdots$ \\
\hline SDSS J095122.57+263513.9 & 1.246 & 17.23 & 1.08 & 1.10 & $\ldots$ & $\ldots$ & known lens (FBQ0951+2635) & 2 \\
\hline SDSS J095228.95+191014.8 & 0.884 & 18.70 & 0.52 & 1.80 & $\mathrm{Te}(\mathrm{I})$ & $\ldots$ & single QSO & $\cdots$ \\
\hline SDSS J095511.67+194112.2 & 1.362 & 17.87 & 0.97 & 0.05 & $\mathrm{Te}(\mathrm{I})$ & $\cdots$ & no lensing object & $\cdots$ \\
\hline SDSS J095636.42+690028.3 & 1.975 & 17.60 & 0.77 & 0.81 & $\mathrm{Te}(\mathrm{I})$ & $\cdots$ & no lensing object & $\cdots$ \\
\hline SDSS J101232.69+224004.7 & 1.843 & 17.52 & 0.43 & 1.05 & $\mathrm{Te}(\mathrm{I})$ & $\cdots$ & single QSO & $\cdots$ \\
\hline SDSS J105247.46+282310.2 & 0.796 & 18.41 & 0.42 & 0.29 & $\mathrm{Te}(\mathrm{I})$ & $\ldots$ & single QSO & $\ldots$ \\
\hline SDSS J105440.83+273306.4 & 1.451 & 16.80 & 1.20 & 1.80 & $\mathrm{Te}(\mathrm{V}, \mathrm{R}, \mathrm{I})$ & $\mathrm{FO}$ & SDSS lens & 1 \\
\hline SDSS J111107.19+180201.5 & 0.754 & 18.37 & 0.45 & 1.38 & $\mathrm{Te}(\mathrm{I})$ & $\cdots$ & single QSO & $\cdots$ \\
\hline SDSS J112135.13+302254.5 & 0.639 & 18.93 & 0.76 & 1.92 & $\mathrm{Te}(\mathrm{I})$ & $\cdots$ & QSO+galaxy & $\cdots$ \\
\hline SDSS J112137.34+222823.7 & 0.608 & 18.58 & 0.40 & 0.12 & $\mathrm{Te}(\mathrm{I})$ & $\ldots$ & single QSO & $\cdots$ \\
\hline SDSS J112235.03+232634.9 & 1.249 & 19.03 & 0.70 & 0.22 & $\mathrm{Te}(\mathrm{I})$ & $\mathrm{FO}$ & separation 0.7 arcsec & $\cdots$ \\
\hline SDSS J112648.19+204512.7 & 1.118 & 18.89 & 0.42 & 1.92 & $\mathrm{Te}(\mathrm{I})$ & $\cdots$ & single QSO & $\cdots$ \\
\hline SDSS J112818.49+240217.4 & 1.607 & 17.88 & 0.77 & 0.66 & $\mathrm{Te}(\mathrm{V}, \mathrm{I})$ & GM & SDSS lens & 3 \\
\hline SDSS J113217.68+182740.7 & 0.852 & 19.00 & 0.41 & 1.17 & $\mathrm{Te}(\mathrm{I})$ & $\ldots$ & single QSO & $\cdots$ \\
\hline SDSS J113454.49+300526.3 & 0.613 & 18.18 & 0.51 & 2.05 & $\mathrm{Te}(\mathrm{I})$ & $\cdots$ & single QSO & $\cdots$ \\
\hline SDSS J114019.74+205853.4 & 1.789 & 17.85 & 3.21 & 1.05 & $\mathrm{Te}(\mathrm{V}, \mathrm{I})$ & $\ldots$ & no lensing object & $\ldots$ \\
\hline SDSS J120929.39+213323.9 & 1.104 & 18.40 & 0.40 & 1.13 & $\mathrm{Te}(\mathrm{I})$ & $\cdots$ & single QSO & $\cdots$ \\
\hline SDSS J121333.78+233521.1 & 2.105 & 17.36 & 0.46 & 0.91 & $\mathrm{Te}(\mathrm{I})$ & $\cdots$ & single QSO & $\cdots$ \\
\hline SDSS J121724.18+214914.4 & 0.669 & 18.22 & 0.57 & 0.46 & $\mathrm{Te}(\mathrm{I})$ & $\ldots$ & single QSO & $\cdots$ \\
\hline SDSS J122249.86+334750.4 & 0.945 & 17.24 & 0.49 & 1.86 & $\mathrm{Te}(\mathrm{I})$ & $\cdots$ & QSO+galaxy & $\cdots$ \\
\hline
\end{tabular}


Table 3-Continued

\begin{tabular}{|c|c|c|c|c|c|c|c|c|}
\hline Object & Redshift $^{\mathrm{a}}$ & $i^{\mathrm{b}}$ & $\theta_{\mathrm{SDSS}^{\mathrm{c}}}$ & $|\Delta i|^{\mathrm{c}}$ & Image $^{d}$ & Spec $^{\mathrm{d}}$ & Comment & Ref. \\
\hline SDSS J122839.19+035749.2 & 0.607 & 18.31 & 0.49 & 0.64 & $\mathrm{Te}(\mathrm{I})$ & $\cdots$ & single QSO & $\ldots$ \\
\hline SDSS J124257.32+254303.0 & 0.826 & 18.71 & 2.77 & 1.30 & $\mathrm{Te}(\mathrm{I})$ & FO & QSO pair $(\mathrm{z}=0.827,0.824)$ & $\cdots$ \\
\hline SDSS J125107.57+293540.5 & 0.802 & 18.86 & 1.23 & 1.02 & $8 \mathrm{k}(\mathrm{V}, \mathrm{I}), \mathrm{Op}(\mathrm{B}, \mathrm{R}, \mathrm{I})$ & $\cdots$ & SDSS lens & 4 \\
\hline SDSS J130451.52+161138.2 & 0.709 & 19.07 & 0.48 & 1.80 & $\mathrm{Te}(\mathrm{I})$ & $\cdots$ & single QSO & $\cdots$ \\
\hline SDSS J132559.51+144110.3 & 1.102 & 18.71 & 0.62 & 0.64 & $\mathrm{Te}(\mathrm{I})$ & $\cdots$ & no lensing object & $\cdots$ \\
\hline SDSS J132752.04+103627.2 & 1.895 & 18.68 & 0.54 & 1.30 & $\mathrm{Te}(\mathrm{I})$ & $\cdots$ & no lensing object & $\cdots$ \\
\hline SDSS J133018.64+181032.1 & 1.393 & 18.35 & 1.11 & 0.77 & $\mathrm{Te}(\mathrm{V}, \mathrm{I}), \mathrm{NF}(\mathrm{H}), \mathrm{PA}(\mathrm{H}, \mathrm{Ks})$ & $\cdots$ & SDSS lens & 5 \\
\hline SDSS J133243.66+343300.5 & 1.925 & 19.08 & 1.16 & 0.16 & $\mathrm{Te}(\mathrm{I})$ & $\cdots$ & no lensing object & $\cdots$ \\
\hline SDSS J140515.42+095931.3 & 1.810 & 19.05 & 1.84 & 0.64 & $8 \mathrm{k}(\mathrm{V}, \mathrm{I}), \mathrm{Te}(\mathrm{R})$ & DO & SDSS lens & 3,6 \\
\hline SDSS J140901.87+294633.6 & 1.583 & 18.54 & 0.95 & 1.23 & $\mathrm{Te}(\mathrm{V}, \mathrm{I})$ & $\cdots$ & no lensing object & $\cdots$ \\
\hline SDSS J141737.89+293510.0 & 0.835 & 18.55 & 0.63 & 0.02 & $\mathrm{Te}(\mathrm{I})$ & $\cdots$ & no lensing object & $\cdots$ \\
\hline SDSS J142341.94+204001.5 & 0.842 & 18.97 & 1.06 & 1.30 & $\mathrm{FO}(\mathrm{I})$ & FO & QSO pair $(\mathrm{z}=0.842,0.842)$ & $\cdots$ \\
\hline SDSS J143451.86+155624.7 & 1.758 & 18.38 & 0.42 & 0.57 & $\mathrm{Te}(\mathrm{I})$ & $\cdots$ & single QSO & $\cdots$ \\
\hline SDSS J144208.76+260903.7 & 1.418 & 18.31 & 1.72 & 0.79 & $\ldots$ & FO & $\mathrm{QSO}+$ star & $\cdots$ \\
\hline SDSS J144738.24+230034.1 & 1.604 & 18.40 & 1.26 & 1.20 & $\mathrm{Te}(\mathrm{V}, \mathrm{I})$ & $\cdots$ & single QSO & $\cdots$ \\
\hline SDSS J145501.91+144734.8 & 1.424 & 18.22 & 1.72 & 0.70 & $\mathrm{WF}(\mathrm{g}, \mathrm{i}), \mathrm{Te}(\mathrm{V}, \mathrm{R}, \mathrm{I})$ & FO & SDSS lens & 1 \\
\hline SDSS J145816.37+222624.5 & 0.979 & 18.23 & 0.60 & 2.21 & $\mathrm{Te}(\mathrm{I})$ & $\cdots$ & single QSO & $\cdots$ \\
\hline SDSS J150248.48+133631.7 & 2.063 & 18.88 & 1.25 & 0.21 & $\mathrm{Te}(\mathrm{I})$ & $\cdots$ & QSO+galaxy & $\cdots$ \\
\hline SDSS J151538.59+151135.8 & 2.054 & 18.05 & 2.03 & 0.26 & $\mathrm{Te}(\mathrm{V}, \mathrm{R}, \mathrm{I}), \mathrm{SC}(\mathrm{i}), \mathrm{SP}(\mathrm{z}), \mathrm{IR}(\mathrm{K})$ & WF,DO & SDSS lens & 3 \\
\hline SDSS J152024.49+211155.3 & 1.505 & 18.40 & 1.24 & 0.91 & $\mathrm{Te}(\mathrm{V}, \mathrm{R}, \mathrm{I})$ & GM & QSO+star & $\cdots$ \\
\hline SDSS J153417.62+253847.0 & 0.645 & 18.84 & 0.84 & 0.09 & $\mathrm{Te}(\mathrm{V}, \mathrm{R}, \mathrm{I})$ & GM & $\mathrm{QSO}+$ star & $\cdots$ \\
\hline SDSS J154817.88+044101.4 & 1.035 & 18.57 & 1.22 & 0.77 & $\cdots$ & FO & QSO+star & $\cdots$ \\
\hline SDSS J155259.18+230104.9 & 1.357 & 17.71 & 0.83 & 0.03 & $\mathrm{Te}(\mathrm{V}, \mathrm{I})$ & $\cdots$ & no lensing object & $\cdots$ \\
\hline SDSS J161320.01+170839.2 & 1.549 & 18.67 & 0.78 & 0.51 & $\mathrm{Te}(\mathrm{I})$ & $\cdots$ & no lensing object & $\cdots$ \\
\hline SDSS J161512.49+182354.0 & 1.026 & 18.96 & 2.20 & 2.13 & $\mathrm{Te}(\mathrm{I})$ & $\cdots$ & QSO+galaxy & $\cdots$ \\
\hline SDSS J161848.15+171459.4 & 1.247 & 19.04 & 1.15 & 1.42 & $\mathrm{Te}(\mathrm{I})$ & $\cdots$ & no lensing object & $\cdots$ \\
\hline
\end{tabular}




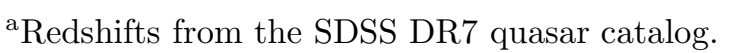

${ }^{\mathrm{b}} i$-band PSF magnitudes with Galactic extinction corrections from the SDSS DR7 quasar catalog.

${ }^{\mathrm{C}}$ Image separations $\left(\theta_{\mathrm{SDSS}}\right)$ in units of arcsec and absolute $i$-band magnitude differences $(|\Delta i|)$ between the expected two components, derived from fitting the SDSS $i$-band image of each candidate with two PSFs using GALFIT.

${ }^{\mathrm{d}}$ Instruments (and filters) used for the follow-up observations. Te: Tek2k CCD at UH88, Op: Optic CCD at UH88, 8k: UH8k at UH88, WF: WFGS2 at UH88, FO: FOCAS at Subaru, , SC: Suprime-Cam at Subaru, IR: IRCS at Subaru, SP: SPIcam at ARC 3.5m, NF: NICFPS at ARC 3.5m, GM: GMOS at Gemini North, PA: PANIC at WB 6.5m, DO: DOLORES at TNG $3.6 \mathrm{~m}$.

References. - (1) Kavo et al. 2010; (2) Schechter et al. 1998; (3) N. Inada, et al., in preparation; (4) Kavo et al. 2007; (5) Oguri et al. 2008d; (6) Jackson et al. 2012 
Table 4. DR7 Color Selected Candidates

\begin{tabular}{|c|c|c|c|c|c|c|c|}
\hline Object & Redshift $^{\mathrm{a}}$ & $i^{\mathrm{b}}$ & $\theta_{\mathrm{SDSS}}{ }^{\mathrm{c}}$ & Image $^{\mathrm{d}}$ & Spec $^{\mathrm{d}}$ & Comment & Ref. \\
\hline SDSS J080713.57+155844.5 & 2.159 & 18.97 & & & & & \\
\hline SDSS J080713.05+155838.5 & $\ldots$ & 19.70 & 9.72 & $\mathrm{Te}(\mathrm{I})$ & $\ldots$ & no lensing object & $\ldots$ \\
\hline SDSS J080753.56+131505.7 & 1.131 & 18.27 & & & & & \\
\hline SDSS J080753.74+131505.5 & $\ldots$ & 19.53 & 2.47 & $\mathrm{Te}(\mathrm{I})$ & $\ldots$ & QSO+galaxy & $\ldots$ \\
\hline SDSS J081753.35+125821.5 & 1.975 & 18.77 & & & & & \\
\hline SDSS J081754.04+125836.0 & 1.700 & 19.49 & 17.57 & $\ldots$ & DA & QSO pair & $\cdots$ \\
\hline SDSS J082234.33+170935.9 & 1.780 & 18.39 & & & & & \\
\hline SDSS J082234.38+170955.0 & 1.798 & 19.65 & 19.02 & $\cdots$ & DA & QSO pair & $\cdots$ \\
\hline SDSS J082911.62+114008.7 & 1.476 & 18.99 & & & & & \\
\hline SDSS J082910.61+114012.1 & 0.802 & 18.52 & 15.23 & $\cdots$ & DA & QSO pair & $\cdots$ \\
\hline SDSS J083805.87+145152.2 & 0.981 & 19.04 & & & & & \\
\hline SDSS J083805.69+145152.7 & $\cdots$ & 20.26 & 2.64 & $\mathrm{Te}(\mathrm{V}, \mathrm{I})$ & $\cdots$ & no lensing object & $\cdots$ \\
\hline SDSS J084629.79+161729.4 & 1.858 & 18.99 & & & & & \\
\hline SDSS J084629.63+161727.1 & $\ldots$ & 19.94 & 3.26 & $\mathrm{Te}(\mathrm{V}, \mathrm{I})$ & $\ldots$ & QSO+galaxy & $\ldots$ \\
\hline SDSS J084836.45+571917.0 & 1.958 & 18.96 & & & & & \\
\hline SDSS J084836.78+571917.2 & $\ldots$ & 20.18 & 2.72 & $\mathrm{Te}(\mathrm{I})$ & $\ldots$ & QSO+galaxy & $\ldots$ \\
\hline SDSS J085139.53+155300.3 & 1.590 & 18.69 & & & & & \\
\hline SDSS J085139.69+155301.1 & $\ldots$ & 19.61 & 2.36 & $\mathrm{Te}(\mathrm{I})$ & $\ldots$ & QSO+galaxy & $\ldots$ \\
\hline SDSS J090327.11+194842.2 & 0.743 & 18.89 & & & & & \\
\hline SDSS J090326.75+194844.2 & $\ldots$ & 19.58 & 5.53 & . & $\mathrm{DA}$ & $\mathrm{QSO}+$ star & $\ldots$ \\
\hline SDSS J090437.50+113427.7 & 0.918 & 19.00 & & & & & \\
\hline SDSS J090437.41+113426.2 & $\cdots$ & 18.74 & 2.06 & $\mathrm{Te}(\mathrm{I})$ & $\cdots$ & no lensing object & $\ldots$ \\
\hline SDSS J091146.01+235112.6 & 1.463 & 18.82 & & & & & \\
\hline SDSS J091146.17+235114.2 & $\ldots$ & 19.54 & 2.61 & $\mathrm{Te}(\mathrm{I})$ & $\ldots$ & no lensing object & $\cdots$ \\
\hline SDSS J091655.54+193437.8 & 1.666 & 18.98 & & & & & \\
\hline SDSS J091656.42+193429.3 & $\cdots$ & 19.96 & 15.00 & $\mathrm{Te}(\mathrm{I})$ & WF & QSO+unknown(not QSO) & $\cdots$ \\
\hline SDSS J091808.86+243550.0 & 1.218 & 18.52 & & & & & \\
\hline SDSS J091809.07+243604.0 & 1.223 & 19.60 & 14.22 & $\operatorname{Te}(\mathrm{I})$ & DA,DO & QSO pair & $\cdots$ \\
\hline
\end{tabular}


Table 4-Continued

\begin{tabular}{|c|c|c|c|c|c|c|c|}
\hline Object & Redshift $^{\mathrm{a}}$ & $i^{\mathrm{b}}$ & $\theta_{\mathrm{SDSS}}{ }^{\mathrm{c}}$ & Image $^{\mathrm{d}}$ & $\operatorname{Spec}^{\mathrm{d}}$ & Comment & Ref. \\
\hline SDSS J093230.57+161008.5 & 1.918 & 18.86 & & & & & \\
\hline SDSS J093230.73+161006.2 & $\cdots$ & 19.91 & 3.32 & $\mathrm{Te}(\mathrm{I})$ & $\cdots$ & no lensing object & $\cdots$ \\
\hline SDSS J094234.97+231031.1 & 1.833 & 18.99 & & & & & \\
\hline SDSS J094235.04+231028.9 & 1.833 & 19.70 & 2.45 & $8 \mathrm{k}(\mathrm{R}, \mathrm{I}), \mathrm{NF}(\mathrm{K})$ & MS & QSO pair & $\ldots$ \\
\hline SDSS J094316.97+644333.1 & 1.549 & 19.07 & & & & & \\
\hline SDSS J094316.77+644335.3 & $\cdots$ & 19.61 & 2.55 & $\mathrm{Te}(\mathrm{V}, \mathrm{I})$ & $\cdots$ & QSO+galaxy & $\cdots$ \\
\hline SDSS J095106.40+195537.6 & 1.412 & 18.72 & & & & & \\
\hline SDSS J095106.39+195534.3 & $\cdots$ & 19.53 & 3.37 & $\mathrm{Te}(\mathrm{V}, \mathrm{I})$ & $\cdots$ & QSO+galaxy & $\cdots$ \\
\hline SDSS J095256.20+222651.0 & 1.537 & 18.34 & & & & & \\
\hline SDSS J095256.25+222636.3 & $\cdots$ & 19.01 & 14.84 & . & DA & $\mathrm{QSO}+$ star & $\cdots$ \\
\hline SDSS J095657.64+212818.6 & 1.548 & 18.11 & & & & & \\
\hline SDSS J095657.83+212759.0 & $\ldots$ & 19.08 & 19.92 & $\mathrm{Te}(\mathrm{I})$ & $\ldots$ & no lensing object & $\ldots$ \\
\hline SDSS J100817.27+141908.6 & 1.513 & 19.08 & & & & & \\
\hline SDSS J100817.89+141909.1 & $\cdots$ & 19.47 & 8.93 & $\mathrm{Te}(\mathrm{I})$ & . & no lensing object & $\cdots$ \\
\hline SDSS J100941.34+250104.0 & 1.873 & 18.82 & & & & & \\
\hline SDSS J100940.58+250053.9 & 1.982 & 19.56 & 14.59 & $\ldots$ & DA & QSO pair & $\ldots$ \\
\hline SDSS J101344.23+285043.5 & 1.559 & 18.98 & & & & & \\
\hline SDSS J101344.17+285038.1 & $\cdots$ & 19.74 & 5.53 & $\mathrm{Te}(\mathrm{I})$ & . & no lensing object & $\cdots$ \\
\hline SDSS J102143.28+193425.4 & 1.436 & 18.87 & & & & & \\
\hline SDSS J102143.57+193421.0 & 1.870 & 20.01 & 6.01 & $\ldots$ & DA & QSO pair & $\ldots$ \\
\hline SDSS J102631.99+250830.2 & 1.936 & 19.02 & & & & & \\
\hline SDSS J102630.61+250836.0 & $\cdots$ & 19.67 & 19.63 & $\cdots$ & DA & $\mathrm{QSO}+$ star & $\cdots$ \\
\hline SDSS J104048.76+263444.0 & 1.832 & 18.82 & & & & & \\
\hline SDSS J104050.14+263446.5 & $\cdots$ & 20.03 & 18.57 & . & DA & QSO+star & . \\
\hline SDSS J104143.09+195722.1 & 1.843 & 18.81 & & & & & \\
\hline SDSS J104143.86+195724.4 & 2.144 & 19.31 & 10.91 & $\ldots$ & DA & QSO pair & $\cdots$ \\
\hline SDSS J104705.64+231437.8 & 1.599 & 18.87 & & & & & \\
\hline SDSS J104705.26+231419.7 & $\cdots$ & 19.65 & 18.93 & $\mathrm{Te}(\mathrm{I})$ & $\mathrm{WF}$ & QSO+star & $\cdots$ \\
\hline
\end{tabular}


Table 4-Continued

\begin{tabular}{|c|c|c|c|c|c|c|c|}
\hline Object & Redshift $^{\mathrm{a}}$ & $i^{\mathrm{b}}$ & $\theta_{\mathrm{SDSS}}{ }^{\mathrm{c}}$ & Image $^{d}$ & $\operatorname{Spec}^{\mathrm{d}}$ & Comment & Ref. \\
\hline SDSS J104836.68+213843.9 & 1.856 & 19.04 & & & & & \\
\hline SDSS J104836.59+213823.9 & $\ldots$ & 18.26 & 20.09 & $\mathrm{Te}(\mathrm{I})$ & . & no lensing object & . \\
\hline SDSS J105406.95+315644.6 & 1.389 & 18.88 & & & & & \\
\hline SDSS J105407.57+315659.2 & $\ldots$ & 19.74 & 16.55 & $\mathrm{Te}(\mathrm{I})$ & $\cdots$ & no lensing object & $\ldots$ \\
\hline SDSS J110049.03+205129.7 & 2.161 & 18.88 & & & & & \\
\hline SDSS J110049.81+205135.2 & $\cdots$ & 17.97 & 12.03 & $\mathrm{Te}(\mathrm{I})$ & $\cdots$ & no lensing object & $\cdots$ \\
\hline SDSS J110502.55+183115.2 & 1.139 & 18.45 & & & & & \\
\hline SDSS J110502.43+183114.5 & $\cdots$ & 19.68 & 1.96 & $\mathrm{Te}(\mathrm{I})$ & $\cdots$ & QSO+galaxy & $\cdots$ \\
\hline SDSS J110807.90+330611.2 & 1.508 & 18.75 & & & & & \\
\hline SDSS J110808.29+330608.9 & 2.340 & 19.67 & 5.41 & $\ldots$ & DA & QSO pair & $\ldots$ \\
\hline SDSS J111427.40+232729.2 & 1.111 & 18.67 & & & & & \\
\hline SDSS J111427.30+232730.2 & $\ldots$ & 19.84 & 1.71 & $\mathrm{Te}(\mathrm{I})$ & . & no lensing object & .. \\
\hline SDSS J112713.53+170954.8 & 1.501 & 18.98 & & & & & \\
\hline SDSS J112713.18+171011.9 & $\cdots$ & 20.03 & 17.82 & $\mathrm{WF}(\mathrm{i})$ & $\cdots$ & no lensing object & $\cdots$ \\
\hline SDSS J113120.92+172217.9 & 1.522 & 18.74 & & & & & \\
\hline SDSS J113121.26+172159.7 & $\ldots$ & 20.01 & 18.83 & WF(i) & .. & no lensing object & $\ldots$ \\
\hline SDSS J113736.00+194324.3 & 1.623 & 18.87 & & & & & \\
\hline SDSS J113737.00+194316.5 & $\ldots$ & 19.41 & 16.05 & $\ldots$ & DA & QSO+star & $\cdots$ \\
\hline SDSS J113933.12+261319.9 & 0.720 & 18.93 & & & & & \\
\hline SDSS J113933.61+261326.7 & $\ldots$ & 17.68 & 9.37 & $\cdots$ & DA,DO & QSO+star & $\cdots$ \\
\hline SDSS J115844.67+272356.9 & 2.156 & 18.46 & & & & & \\
\hline SDSS J115843.70+272354.5 & $\ldots$ & 17.59 & 13.27 & 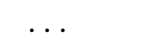 & WF & $\mathrm{QSO}+$ star & \\
\hline SDSS J123316.04+174854.8 & 2.187 & 19.03 & & & & & \\
\hline SDSS J123315.30+174908.8 & 1.643 & 20.29 & 17.50 & $\cdots$ & $\mathrm{DA}$ & QSO pair & $\cdots$ \\
\hline SDSS J124300.48+204246.7 & 1.980 & 18.02 & & & & & \\
\hline SDSS J124300.33+204246.8 & $\cdots$ & 19.12 & 2.13 & $\mathrm{Te}(\mathrm{V}, \mathrm{I})$ & . & QSO+galaxy & $\cdots$ \\
\hline SDSS J125022.32+174144.5 & 1.246 & 19.06 & & & & & \\
\hline SDSS J125023.29+174142.3 & 1.241 & 18.63 & 13.91 & $\mathrm{Te}(\mathrm{I})$ & WF & QSO pair & $\cdots$ \\
\hline
\end{tabular}


Table 4-Continued

\begin{tabular}{|c|c|c|c|c|c|c|c|}
\hline Object & Redshift $^{\mathrm{a}}$ & $i^{\mathrm{b}}$ & $\theta_{\mathrm{SDSS}^{\mathrm{c}}}$ & Image $^{\mathrm{d}}$ & $\operatorname{Spec}^{\mathrm{d}}$ & Comment & Ref. \\
\hline SDSS J125150.47+250438.4 & 1.526 & 18.81 & & & & & \\
\hline SDSS J125150.46+250452.8 & $\ldots$ & 17.61 & 14.30 & $\mathrm{Te}(\mathrm{I})$ & . & no lensing object & $\ldots$ \\
\hline SDSS J125313.42+101926.4 & 0.716 & 17.47 & & & & & \\
\hline SDSS J125313.18+101914.1 & $\ldots$ & 18.39 & 12.95 & $\ldots$ & DA & $\mathrm{QSO}+$ star & $\ldots$ \\
\hline SDSS J130424.98+353326.0 & 1.988 & 18.17 & & & & & \\
\hline SDSS J130424.79+353337.7 & $\cdots$ & 18.15 & 11.79 & $\cdots$ & DA & QSO+star & $\cdots$ \\
\hline SDSS J130439.13+210559.9 & 2.015 & 18.77 & & & & & \\
\hline SDSS J130438.38+210555.4 & $\cdots$ & 18.25 & 11.60 & $\mathrm{Te}(\mathrm{I})$ & $\cdots$ & no lensing object & $\cdots$ \\
\hline SDSS J130548.06+261930.0 & 1.496 & 19.09 & & & & & \\
\hline SDSS J130548.83+261932.7 & $\ldots$ & 18.21 & 10.67 & $\mathrm{Te}(\mathrm{I})$ & $\ldots$ & no lensing object & $\ldots$ \\
\hline SDSS J131017.23+105448.3 & 2.134 & 18.68 & & & & & \\
\hline SDSS J131018.17+105458.3 & $\ldots$ & 19.00 & 16.93 & $\mathrm{Te}(\mathrm{I})$ & . & no lensing object & . \\
\hline SDSS J131531.76+262132.3 & 1.525 & 18.45 & & & & & \\
\hline SDSS J131531.82+262116.2 & $\cdots$ & 19.08 & 16.14 & $\mathrm{Te}(\mathrm{I})$ & $\cdots$ & no lensing object & $\cdots$ \\
\hline SDSS J131847.14+105245.0 & 0.844 & 19.02 & & & & & \\
\hline SDSS J131846.35+105233.6 & 1.910 & 19.16 & 16.41 & $\mathrm{Te}(\mathrm{I})$ & WF & QSO pair & $\ldots$ \\
\hline SDSS J132059.17+164402.5 & 1.502 & 18.87 & & & & & \\
\hline SDSS J132059.73+164405.6 & 1.502 & 18.61 & 8.59 & $\mathrm{Te}(\mathrm{V}, \mathrm{I}), \mathrm{MO}(\mathrm{J}, \mathrm{H}, \mathrm{K})$ & DA & SDSS lens & 1 \\
\hline SDSS J132551.85+080957.2 & 1.448 & 18.71 & & & & & \\
\hline SDSS J132551.79+080947.0 & $\ldots$ & 18.01 & 10.32 & $\mathrm{Te}(\mathrm{I})$ & $\cdots$ & no lensing object & $\ldots$ \\
\hline SDSS J133139.33+082647.1 & 1.586 & 17.98 & & & & & \\
\hline SDSS J133138.87+082641.1 & $\ldots$ & 19.29 & 9.15 & $\mathrm{Te}(\mathrm{I})$ & . & no lensing object & $\cdots$ \\
\hline SDSS J133353.11+132614.0 & 1.786 & 18.86 & & & & & \\
\hline SDSS J133353.20+132612.0 & $\cdots$ & 20.02 & 2.35 & $\mathrm{Te}(\mathrm{I})$ & $\ldots$ & no lensing object & $\cdots$ \\
\hline SDSS J133818.02+095210.8 & 1.690 & 18.61 & & & & & \\
\hline SDSS J133818.01+095212.3 & $\cdots$ & 19.61 & 1.50 & $\mathrm{Te}(\mathrm{V}, \mathrm{I})$ & .. & no lensing object & $\cdots$ \\
\hline SDSS J134953.90+220927.7 & 1.308 & 18.88 & & & & & \\
\hline SDSS J134953.79+220912.5 & $\ldots$ & 19.35 & 15.25 & $\mathrm{Te}(\mathrm{I})$ & $\cdots$ & no lensing object & $\cdots$ \\
\hline
\end{tabular}


Table 4-Continued

\begin{tabular}{|c|c|c|c|c|c|c|c|}
\hline Object & Redshift $^{\mathrm{a}}$ & $i^{\mathrm{b}}$ & $\theta_{\mathrm{SDSS}^{\mathrm{c}}}$ & Image $^{\mathrm{d}}$ & Spec $^{\mathrm{d}}$ & Comment & Ref. \\
\hline SDSS J135020.67+190337.8 & 1.261 & 18.40 & & & & & \\
\hline SDSS J135020.52+190340.2 & $\ldots$ & 19.13 & 3.12 & $\mathrm{Te}(\mathrm{I})$ & $\ldots$ & no lensing object & $\cdots$ \\
\hline SDSS J135302.69+230259.8 & 1.542 & 18.74 & & & & & \\
\hline SDSS J135303.07+230309.8 & $\ldots$ & 19.83 & 11.18 & $\mathrm{Te}(\mathrm{I})$ & $\ldots$ & no lensing object & $\ldots$ \\
\hline SDSS J135455.03+072926.8 & 0.872 & 18.80 & & & & & \\
\hline SDSS J135455.24+072909.9 & 1.351 & 19.35 & 17.18 & $\mathrm{Te}(\mathrm{I})$ & WF & QSO pair & $\cdots$ \\
\hline SDSS J135530.93+195808.1 & 0.801 & 18.87 & & & & & \\
\hline SDSS J135531.07+195804.2 & $\cdots$ & 19.27 & 4.31 & $\mathrm{Te}(\mathrm{I})$ & $\cdots$ & no lensing object & $\cdots$ \\
\hline SDSS J135607.71+162044.9 & 1.184 & 18.95 & & & & & \\
\hline SDSS J135607.84+162045.5 & $\ldots$ & 19.90 & 1.85 & $\mathrm{WF}(\mathrm{g}, \mathrm{i})$ & FO & $\mathrm{QSO}+$ star & $\ldots$ \\
\hline SDSS J135809.86+232610.0 & 1.555 & 18.91 & & & & & \\
\hline SDSS J135810.68+232604.5 & 1.543 & 19.91 & 12.46 & $\ldots$ & DA & QSO pair & . \\
\hline SDSS J140012.27+232346.7 & 1.877 & 18.34 & & & & & \\
\hline SDSS J140012.86+232351.9 & 1.867 & 19.27 & 9.50 & $\ldots$ & DA & QSO pair & $\cdots$ \\
\hline SDSS J140914.66+163054.4 & 1.223 & 18.84 & & & & & \\
\hline SDSS J140914.39+163058.6 & 0.303 & 17.96 & 5.78 & $\ldots$ & DA & QSO pair & $\ldots$ \\
\hline SDSS J141023.94+315953.5 & 1.181 & 18.85 & & & & & \\
\hline SDSS J141024.13+315952.5 & $\ldots$ & 19.87 & 2.61 & $\mathrm{Te}(\mathrm{I})$ & $\ldots$ & no lensing object & $\ldots$ \\
\hline SDSS J141118.48+083945.3 & 1.143 & 18.66 & & & & & \\
\hline SDSS J141118.83+083943.8 & $\ldots$ & 18.36 & 5.37 & $\mathrm{Te}(\mathrm{I})$ & $\ldots$ & no lensing object & $\ldots$ \\
\hline SDSS J141302.61+194938.0 & 1.540 & 18.55 & & & & & \\
\hline SDSS J141303.36+194940.2 & $\ldots$ & 18.88 & 10.70 & $\mathrm{Te}(\mathrm{I})$ & $\cdots$ & no lensing object & 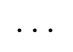 \\
\hline SDSS J142054.41+160333.3 & 2.021 & 18.97 & & & & & \\
\hline SDSS J142054.91+160343.0 & 2.066 & 19.53 & 11.96 & $\mathrm{Te}(\mathrm{I})$ & GM & QSO pair & $\cdots$ \\
\hline SDSS J142630.23+252743.2 & 1.922 & 18.59 & & & & & \\
\hline SDSS J142629.27+252732.0 & $\ldots$ & 18.41 & 17.22 & $\mathrm{Te}(\mathrm{I})$ & $\cdots$ & no lensing object & $\cdots$ \\
\hline SDSS J142931.29+261654.1 & 1.403 & 18.18 & & & & & \\
\hline SDSS J142931.17+261638.5 & $\ldots$ & 19.11 & 15.73 & $\mathrm{Te}(\mathrm{I})$ & $\ldots$ & no lensing object & $\ldots$ \\
\hline
\end{tabular}


Table 4-Continued

\begin{tabular}{|c|c|c|c|c|c|c|c|}
\hline Object & Redshift $^{\mathrm{a}}$ & $i^{\mathrm{b}}$ & $\theta_{\mathrm{SDSS}}{ }^{\mathrm{c}}$ & Image $^{\mathrm{d}}$ & $\operatorname{Spec}^{\mathrm{d}}$ & Comment & Ref. \\
\hline SDSS J143002.88+071411.3 & 1.258 & 19.01 & & & & & \\
\hline SDSS J143002.66+071415.6 & 1.246 & 19.68 & 5.41 & $\ldots$ & $\ldots$ & QSO pair (see reference) & 2 \\
\hline SDSS J143226.30+080730.4 & 1.729 & 18.94 & & & & & \\
\hline SDSS J143226.44+080741.2 & 2.125 & 19.87 & 10.97 & $\ldots$ & DA & QSO pair & $\ldots$ \\
\hline SDSS J143350.94+145008.1 & 1.506 & 18.82 & & & & & \\
\hline SDSS J143351.09+145005.6 & 1.506 & 19.19 & 3.34 & $\mathrm{Te}(\mathrm{I})$ & DA & QSO pair & $\cdots$ \\
\hline SDSS J143553.39+185403.2 & 1.168 & 19.09 & & & & & \\
\hline SDSS J143552.81+185404.0 & $\cdots$ & 20.31 & 8.40 & $\mathrm{Te}(\mathrm{I})$ & GM & QSO+star & $\cdots$ \\
\hline SDSS J144035.91+224023.2 & 1.599 & 18.37 & & & & & \\
\hline SDSS J144036.12+224028.8 & $\ldots$ & 17.99 & 6.26 & $\mathrm{Te}(\mathrm{I})$ & . & no lensing object & $\ldots$ \\
\hline SDSS J144144.92+225808.9 & 2.193 & 18.92 & & & & & \\
\hline SDSS J144145.80+225754.9 & $\ldots$ & 20.16 & 18.54 & $\ldots$ & DA & $\mathrm{QSO}+$ star & $\ldots$ \\
\hline SDSS J144147.28+223346.3 & 1.458 & 18.97 & & & & & \\
\hline SDSS J144147.68+223328.5 & $\ldots$ & 19.45 & 18.60 & $\mathrm{Te}(\mathrm{I})$ & . & no lensing object & $\cdots$ \\
\hline SDSS J145030.61+214818.1 & 2.090 & 18.99 & & & & & \\
\hline SDSS J145031.34+214804.1 & $\cdots$ & 19.54 & 17.33 & $\mathrm{Te}(\mathrm{I}), \mathrm{WF}(\mathrm{i})$ & $\cdots$ & no lensing object & $\cdots$ \\
\hline SDSS J145103.50+221551.0 & 1.509 & 18.74 & & & & & \\
\hline SDSS J145103.92+221536.3 & 1.554 & 19.51 & 15.83 & . & DA & QSO pair & $\cdots$ \\
\hline SDSS J145224.60+090424.2 & 1.873 & 18.73 & & & & & \\
\hline SDSS J145224.19+090436.3 & $\ldots$ & 19.04 & 13.56 & $\mathrm{Te}(\mathrm{I})$ & $\ldots$ & no lensing object & $\ldots$ \\
\hline SDSS J145517.56+282126.0 & 0.867 & 18.92 & & & & & \\
\hline SDSS J145518.58+282117.2 & $\ldots$ & 19.27 & 16.15 & $\mathrm{Te}(\mathrm{I})$ & WF & QSO+star & $\ldots$ \\
\hline SDSS J145529.98+215352.6 & 1.306 & 18.83 & & & & & \\
\hline SDSS J145528.78+215403.3 & $\ldots$ & 18.25 & 19.85 & $\mathrm{Te}(\mathrm{I})$ & WF & QSO+unknown(not QSO) & $\cdots$ \\
\hline SDSS J150429.73+070619.8 & 2.116 & 19.05 & & & & & \\
\hline SDSS J150430.23+070626.6 & $\cdots$ & 17.89 & 10.01 & $\mathrm{Te}(\mathrm{I})$ & $\cdots$ & no lensing object & $\cdots$ \\
\hline SDSS J150506.13+080604.5 & 1.249 & 18.43 & & & & & \\
\hline SDSS J150506.26+080623.2 & 2.154 & 19.37 & 18.81 & & DO & QSO pair & . \\
\hline
\end{tabular}


Table 4-Continued

\begin{tabular}{|c|c|c|c|c|c|c|c|}
\hline Object & Redshift $^{\mathrm{a}}$ & $i^{\mathrm{b}}$ & $\theta_{\mathrm{SDSS}^{\mathrm{c}}}$ & Image $^{\mathrm{d}}$ & $\operatorname{Spec}^{\mathrm{d}}$ & Comment & Ref. \\
\hline SDSS J151245.20+092939.8 & 1.638 & 18.12 & & & & & \\
\hline SDSS J151245.47+092935.7 & .. & 19.28 & 5.72 & $\mathrm{Te}(\mathrm{I})$ & $\ldots$ & no lensing object & $\ldots$ \\
\hline SDSS J152050.18+263740.8 & 1.365 & 19.00 & & & & & \\
\hline SDSS J152050.04+263740.9 & $\ldots$ & 19.12 & 1.94 & $\mathrm{Te}(\mathrm{I})$ & FO & $\mathrm{QSO}+$ star & 3 \\
\hline SDSS J152100.33+063653.5 & 1.555 & 18.65 & & & & & \\
\hline SDSS J152059.46+063659.5 & $\cdots$ & 18.14 & 14.36 & $\mathrm{Te}(\mathrm{I})$ & DA & $\mathrm{QSO}+$ star & $\cdots$ \\
\hline SDSS J152105.50+165424.2 & 1.551 & 19.00 & & & & & \\
\hline SDSS J152105.05+165440.7 & $\cdots$ & 20.25 & 17.70 & $\mathrm{WF}(\mathrm{i})$ & $\cdots$ & no lensing object & $\cdots$ \\
\hline SDSS J152118.34+242555.8 & 2.188 & 18.31 & & & & & \\
\hline SDSS J152117.47+242606.5 & $\ldots$ & 17.29 & 16.04 & $\mathrm{Te}(\mathrm{I})$ & $\ldots$ & no lensing object & $\ldots$ \\
\hline SDSS J152122.41+092650.8 & 0.748 & 18.67 & & & & & \\
\hline SDSS J152122.36+092654.4 & $\ldots$ & 18.84 & 3.65 & $\mathrm{Te}(\mathrm{I})$ & $\cdots$ & no lensing object & .. \\
\hline SDSS J152310.55+175410.2 & 2.122 & 19.01 & & & & & \\
\hline SDSS J152310.10+175352.2 & $\cdots$ & 19.91 & 19.17 & $\mathrm{Te}(\mathrm{I})$ & GM & $\mathrm{QSO}+$ star & $\cdots$ \\
\hline SDSS J152501.48+262732.3 & 1.581 & 18.93 & & & & & \\
\hline SDSS J152500.33+262737.5 & $\cdots$ & 19.88 & 16.33 & $\mathrm{WF}(\mathrm{i})$ & $\cdots$ & no lensing object & $\ldots$ \\
\hline SDSS J152719.52+264929.3 & 0.780 & 19.08 & & & & & \\
\hline SDSS J152719.45+264934.1 & $\ldots$ & 18.55 & 4.90 & $\mathrm{Te}(\mathrm{I})$ & $\ldots$ & no lensing object & $\ldots$ \\
\hline SDSS J152806.63+132345.8 & 0.675 & 18.70 & & & & & \\
\hline SDSS J152806.65+132346.9 & $\ldots$ & 18.40 & 1.13 & $\mathrm{Te}(\mathrm{I})$ & $\cdots$ & no lensing object & $\ldots$ \\
\hline SDSS J153019.08+191948.5 & 1.294 & 18.96 & & & & & \\
\hline SDSS J153020.30+191942.5 & 1.465 & 19.45 & 18.16 & $\mathrm{Te}(\mathrm{I})$ & WF & QSO pair & $\ldots$ \\
\hline SDSS J153043.60+254909.6 & 1.490 & 19.02 & & & & & \\
\hline SDSS J153043.62+254907.1 & $\cdots$ & 20.16 & 2.52 & $\mathrm{Te}(\mathrm{I})$ & $\cdots$ & no lensing object & $\ldots$ \\
\hline SDSS J153254.75+092454.9 & 2.123 & 18.89 & & & & & \\
\hline SDSS J153255.31+092508.0 & $\cdots$ & 18.94 & 15.35 & $\mathrm{WF}(\mathrm{i})$ & $\ldots$ & no lensing object & $\cdots$ \\
\hline SDSS J153444.54+015249.4 & 1.064 & 18.29 & & & & & \\
\hline SDSS J153444.61+015241.7 & $\ldots$ & 17.17 & 7.78 & $\mathrm{Te}(\mathrm{I})$ & WF & $\mathrm{QSO}+$ star & $\ldots$ \\
\hline
\end{tabular}


Table 4-Continued

\begin{tabular}{|c|c|c|c|c|c|c|c|}
\hline Object & Redshift $^{\mathrm{a}}$ & $i^{\mathrm{b}}$ & $\theta_{\mathrm{SDSS}}{ }^{\mathrm{c}}$ & Image $^{\mathrm{d}}$ & Spec $^{\mathrm{d}}$ & Comment & Ref. \\
\hline SDSS J153452.12+093251.0 & 1.168 & 18.82 & & & & & \\
\hline SDSS J153451.37+093248.3 & $\ldots$ & 17.69 & 11.47 & $\mathrm{Te}(\mathrm{I})$ & $\mathrm{DA}$ & QSO+star & $\ldots$ \\
\hline SDSS J153530.86+131310.6 & 1.018 & 18.55 & & & & & \\
\hline SDSS J153531.01+131309.8 & $\ldots$ & 19.81 & 2.35 & $\mathrm{Te}(\mathrm{I})$ & $\ldots$ & no lensing object & $\ldots$ \\
\hline SDSS J153920.08+150902.1 & 1.474 & 18.62 & & & & & \\
\hline SDSS J153919.60+150916.2 & $\cdots$ & 19.39 & 15.69 & $\mathrm{Te}(\mathrm{I})$ & $\mathrm{DA}$ & QSO+star & $\cdots$ \\
\hline SDSS J153927.07+042518.1 & 0.739 & 18.38 & & & & & \\
\hline SDSS J153926.96+042519.1 & $\cdots$ & 18.36 & 1.89 & $\mathrm{Te}(\mathrm{I})$ & $\cdots$ & no lensing object & $\cdots$ \\
\hline SDSS J154043.50+154416.1 & 1.577 & 18.18 & & & & & \\
\hline SDSS J154043.77+154400.5 & $\ldots$ & 18.52 & 16.06 & $\mathrm{Te}(\mathrm{I})$ & $\ldots$ & no lensing object & $\ldots$ \\
\hline SDSS J154356.31+021344.0 & 1.121 & 18.79 & & & & & \\
\hline SDSS J154356.82+021349.2 & $\ldots$ & 19.87 & 9.22 & $\mathrm{Te}(\mathrm{I}), \mathrm{WF}(\mathrm{i})$ & $\ldots$ & no lensing object & $\ldots$ \\
\hline SDSS J154405.23+143455.2 & 1.406 & 17.94 & & & & & \\
\hline SDSS J154405.08+143447.9 & $\cdots$ & 18.36 & 7.66 & $\mathrm{Te}(\mathrm{I}), \mathrm{WF}(\mathrm{i})$ & $\cdots$ & no lensing object & $\ldots$ \\
\hline SDSS J154503.01+164234.4 & 1.481 & 18.67 & & & & & \\
\hline SDSS J154501.90+164226.2 & $\cdots$ & 18.69 & 18.02 & $\cdots$ & $\mathrm{DA}$ & QSO+star & $\ldots$ \\
\hline SDSS J154632.34+223637.8 & 1.984 & 18.65 & & & & & \\
\hline SDSS J154632.28+223640.2 & $\ldots$ & 19.76 & 2.47 & $\mathrm{Te}(\mathrm{I})$ & $\ldots$ & no lensing object & $\ldots$ \\
\hline SDSS J154936.47+115833.2 & 1.248 & 18.91 & & & & & \\
\hline SDSS J154936.35+115833.3 & $\ldots$ & 20.00 & 1.96 & $\mathrm{Te}(\mathrm{I})$ & $\ldots$ & no lensing object & $\ldots$ \\
\hline SDSS J155052.40+183655.1 & 1.823 & 18.44 & & & & & \\
\hline SDSS J155052.79+183646.1 & .. & 17.79 & 10.59 & $\mathrm{Te}(\mathrm{I})$ & $\ldots$ & no lensing object & $\ldots$ \\
\hline SDSS J155446.59+040443.2 & 2.157 & 19.08 & & & & & \\
\hline SDSS J155447.32+040428.6 & $\ldots$ & 18.10 & 18.21 & $\mathrm{Te}(\mathrm{I})$ & $\ldots$ & no lensing object & $\ldots$ \\
\hline SDSS J155520.86+075418.8 & 1.464 & 18.09 & & & & & \\
\hline SDSS J155520.96+075418.0 & $\ldots$ & 19.39 & 1.63 & $\mathrm{Te}(\mathrm{I})$ & $\cdots$ & no lensing object & $\ldots$ \\
\hline SDSS J155626.90+103457.3 & 1.902 & 18.68 & & & & & \\
\hline SDSS J155626.26+103443.9 & $\ldots$ & 19.40 & 16.59 & $\mathrm{Te}(\mathrm{I})$ & $\mathrm{DA}$ & QSO+unknown(not QSO) & $\ldots$ \\
\hline
\end{tabular}


Table 4-Continued

\begin{tabular}{|c|c|c|c|c|c|c|c|}
\hline Object & Redshift $^{\mathrm{a}}$ & $i^{\mathrm{b}}$ & $\theta_{\mathrm{SDSS}}^{\mathrm{c}}$ & Image $^{\mathrm{d}}$ & Spec $^{\mathrm{d}}$ & Comment & Ref. \\
\hline SDSS J160734.18+035034.0 & 1.304 & 18.56 & & & & & \\
\hline SDSS J160733.01+035038.7 & $\cdots$ & 18.74 & 18.02 & $\mathrm{WF}(\mathrm{i})$ & $\cdots$ & no lensing object & $\cdots$ \\
\hline SDSS J161914.41+093615.8 & 1.630 & 17.91 & & & & & \\
\hline SDSS J161914.26+093625.0 & $\cdots$ & 17.86 & 9.46 & $\mathrm{Te}(\mathrm{I})$ & $\cdots$ & no lensing object & $\cdots$ \\
\hline SDSS J162026.14+120342.0 & 1.157 & 19.09 & & & & & \\
\hline SDSS J162026.28+120340.3 & 1.158 & 19.37 & 2.57 & $\mathrm{Te}(\mathrm{V}, \mathrm{R}, \mathrm{I}), \mathrm{WF}(\mathrm{i})$ & FO & SDSS lens & 4 \\
\hline SDSS J162033.71+121121.2 & 1.629 & 18.66 & & & & & \\
\hline SDSS J162032.65+121112.8 & $\ldots$ & 18.33 & 17.90 & $\mathrm{Te}(\mathrm{I})$ & $\ldots$ & no lensing object & $\ldots$ \\
\hline SDSS J162033.47+121117.8 & $\ldots$ & 19.53 & 5.11 & $\mathrm{Te}(\mathrm{I})$ & $\ldots$ & no lensing object & $\ldots$ \\
\hline SDSS J162438.27+105106.8 & 1.227 & 19.02 & & & & & \\
\hline SDSS J162438.01+105105.3 & $\cdots$ & 18.99 & 4.28 & $\mathrm{Te}(\mathrm{I})$ & $\ldots$ & no lensing object & $\cdots$ \\
\hline SDSS J162531.13+103110.5 & 1.312 & 18.94 & & & & & \\
\hline SDSS J162530.77+103104.3 & $\cdots$ & 20.04 & 8.18 & $\mathrm{Te}(\mathrm{I})$ & $\cdots$ & no lensing object & $\cdots$ \\
\hline SDSS J163017.91+113725.0 & 1.637 & 18.67 & & & & & \\
\hline SDSS J163018.20+113710.2 & $\ldots$ & 19.06 & 15.45 & $\mathrm{Te}(\mathrm{I})$ & $\ldots$ & no lensing object & $\ldots$ \\
\hline SDSS J163326.49+203014.6 & 1.553 & 18.99 & & & & & \\
\hline SDSS J163326.02+203020.9 & $\cdots$ & 20.13 & 9.18 & $\mathrm{Te}(\mathrm{I})$ & $\mathrm{DA}$ & QSO+unknown(not QSO) & $\cdots$ \\
\hline SDSS J170924.22+352514.2 & 1.843 & 18.94 & & & & & \\
\hline SDSS J170924.32+352514.1 & $\ldots$ & 19.69 & 1.12 & $\mathrm{Te}(\mathrm{I})$ & $\ldots$ & no lensing object & $\ldots$ \\
\hline
\end{tabular}

${ }^{a}$ Redshifts from the SDSS DR7 quasar catalog or follow-up spectroscopy.

${ }^{\mathrm{b}} i$-band PSF magnitudes with Galactic extinction corrections from the SDSS DR7 quasar catalog.

${ }^{\mathrm{c}}$ Image separations $\left(\theta_{\mathrm{SDSS}}\right)$ in units of arcsec between the two components from the SDSS imaging data.

${ }^{\mathrm{d}}$ Instruments (and filters) used for the follow-up observations. Te: Tek2k CCD at UH88, WF: WFGS2 at UH88, 8k: UH8k at UH88, DA: DIS III at ARC 3.5m, NF: NICFPS at ARC 3.5m, SP: SPIcam at ARC 3.5m, DO: DOLORES at TNG 3.6m, 
MS: MMT spectrograph, FO: FOCAS at Subaru, MO: MOIRCS at Subaru GM: GMOS at Gemini North.

References. - (1) C. E. Rusu et al., in preparation; (2) Myers et al. 2008; (3) Myers et al. 2007; (4) Kavo et al. 2010. 
Table 5. DR7 Statistical Sample

\begin{tabular}{|c|c|c|c|c|c|c|c|c|c|c|}
\hline Object & $N_{\text {img }}$ & $z_{s}^{\mathrm{a}}$ & $z_{l}^{\mathrm{b}}$ & $i^{\mathrm{c}}$ & $\theta_{\max }{ }^{\mathrm{d}}$ & $f_{I} \mathrm{e}$ & $I_{\text {gal }} \mathrm{f}$ & Source & Non-SDSS designation & Ref. \\
\hline SDSS J024634.09-082536.1 & 2 & 1.686 & 0.723 & 17.76 & 1.09 & 0.34 & $20.78(0.04)$ & DR3 & . & $1,2,3$ \\
\hline SDSS J074653.04+440351.3 & 2 & 1.998 & 0.513 & 18.71 & 1.08 & 0.97 & $19.62(0.19)$ & DR5 & $\ldots$ & 4,5 \\
\hline SDSS J080623.70+200631.8 & 2 & 1.538 & 0.573 & 18.89 & 1.49 & 0.76 & $20.16(0.03)$ & DR5 & $\cdots$ & $2,6,7$ \\
\hline SDSS J091301.02+525928.8 & 2 & 1.378 & $(0.830)$ & 16.17 & 1.11 & 0.70 & $18.81(0.45)$ & DR3 & SBS $0909+523$ & $3,8,9$ \\
\hline SDSS J092455.79+021924.9 & 4 & 1.523 & 0.394 & 18.12 & 1.81 & 0.43 & $19.36(0.02)$ & DR3 & $\ldots$ & $3,9,10,11$ \\
\hline SDSS J095122.57+263513.9 & 2 & 1.246 & 0.260 & 17.24 & 1.10 & 0.33 & $19.66(0.03)$ & DR7 & FBQ0951+2635 & $2,3,12$ \\
\hline SDSS J100120.83+555349.6 & 2 & 1.413 & 0.36 & 16.68 & 6.17 & 0.94 & $17.11(0.02)$ & DR3 & Q0957+561 & $3,13,14$ \\
\hline SDSS J100128.61+502756.8 & 2 & 1.841 & 0.415 & 17.32 & 2.86 & 0.86 & $19.63(0.03)$ & DR3 & $\cdots$ & 15 \\
\hline SDSS J100434.92+411242.7 & 5 & 1.740 & 0.68 & 18.82 & 14.72 & 0.23 & $18.82(0.18)$ & DR3 & $\ldots$ & $3,16,17,18,19$ \\
\hline SDSS J102111.01+491330.3 & 2 & 1.720 & $(0.451)$ & 18.99 & 1.14 & 0.43 & $19.82(0.21)$ & DR3 & $\ldots$ & 20 \\
\hline SDSS J105545.45+462839.4 & 2 & 1.249 & 0.388 & 18.76 & 1.15 & 0.33 & $19.73(0.05)$ & DR5 & $\cdots$ & 5 \\
\hline SDSS J111816.94+074558.2 & 4 & 1.735 & 0.311 & 15.97 & 2.43 & 0.25 & $18.91(0.02)$ & DR5 & PG1115+080 & $3,21,22$ \\
\hline SDSS J120629.64+433217.5 & 2 & 1.789 & $(0.748)$ & 18.46 & 2.90 & 0.74 & $19.51(0.03)$ & DR5 & $\cdots$ & 15 \\
\hline SDSS J121646.04+352941.4 & 2 & 2.013 & $\sim 0.55$ & 19.08 & 1.49 & 0.41 & $20.31(0.05)$ & DR5 & $\cdots$ & 23 \\
\hline SDSS J122608.02-000602.2 & 2 & 1.126 & 0.517 & 18.23 & 1.26 & 0.62 & $19.71(0.07)$ & DR3 & $\cdots$ & $3,24,25$ \\
\hline SDSS J125107.57+293540.5 & 4 & 0.802 & 0.410 & 18.86 & 1.79 & 0.36 & $18.43(0.25)$ & DR7 & $\ldots$ & 26 \\
\hline SDSS J131339.98+515128.4 & 2 & 1.877 & 0.194 & 17.70 & 1.24 & 0.92 & $17.49(0.02)$ & DR5 & $\cdots$ & 27 \\
\hline SDSS J132059.17+164402.5 & 2 & 1.502 & $(0.899)$ & 18.88 & 8.59 & 0.73 & $21.59(0.10)$ & DR7 & $\cdots$ & 28 \\
\hline SDSS J133018.64+181032.1 & 4 & 1.393 & 0.373 & 18.35 & 1.76 & 0.33 & $17.84(0.01)$ & DR7 & $\cdots$ & 29 \\
\hline SDSS J133222.62+034739.9 & 2 & 1.438 & 0.191 & 17.89 & 1.14 & 0.70 & $18.06(0.02)$ & DR3 & $\cdots$ & 30 \\
\hline SDSS J133534.79+011805.5 & 2 & 1.571 & 0.440 & 17.54 & 1.63 & 0.37 & $19.40(0.02)$ & DR3 & LBQS1333+0113 & $3,25,31$ \\
\hline SDSS J135306.34+113804.7 & 2 & 1.624 & $\sim 0.25$ & 16.47 & 1.41 & 0.40 & $17.80(0.01)$ & DR5 & $\ldots$ & 6 \\
\hline SDSS J140515.42+095931.3 & 2 & 1.810 & 0.66 & 19.05 & 1.98 & 0.33 & $19.70(0.05)$ & DR7 & ULAS J1405+0959 & 32,33 \\
\hline SDSS J145501.91+144734.8 & 2 & 1.424 & $\sim 0.42$ & 18.22 & 1.73 & 0.50 & $18.51(0.07)$ & DR7 & $\cdots$ & 5 \\
\hline SDSS J151538.59+151135.8 & 2 & 2.054 & $(0.742)$ & 18.05 & 1.95 & 0.64 & $20.02(0.01)$ & DR7 & $\cdots$ & 32 \\
\hline SDSS J152445.62+440949.5 & 2 & 1.210 & 0.320 & 18.76 & 1.67 & 0.56 & $18.33(0.01)$ & DR3 & $\cdots$ & 23 \\
\hline
\end{tabular}


${ }^{a}$ Spectroscopic source redshifts from the SDSS data

${ }^{\mathrm{b}}$ Lens redshifts. Values in parentheses indicate lens redshifts measured from absorption lines in the quasar spectra rather than direct spectroscopy of the lensing galaxy. For lenses without any spectroscopic information, we provide an approximate redshift with an error-bar inferred from the color and magnitude.

${ }^{\mathrm{c}} \mathrm{SDSS}$ PSF magnitude in $i$-band (AB) after correcting for the Galactic extinction.

${ }^{\mathrm{d}}$ Maximum image separations, in units of arcsec, derived from the best imaging data.

${ }^{\mathrm{e}} I$-band flux ratios between the brightest lensed image and the farthest lensed image from the brightest image. Derived from the discovery papers for SQLS lenses, and from the best-quality imaging data (mostly from the HST) for previously known lenses.

${ }^{\mathrm{f}} I$-band (Vega) magnitude of the lensing galaxy without Galactic extinction corrections. Values in parentheses are statistical errors. When the system contains multiple galaxies within the Einstein radius, the magnitude of the brightest galaxy is shown.

${ }^{\mathrm{g}}$ While our analysis indicates that this is a probable gravitational lens system, its lensing nature is not as secure as the other lenses.

References. - (1) Inada et al. 2005b; (2) Eigenbrod et al. 2007; (3) CASTLES webpage (C. S. Kochanek et al., http://cfa-www.harvard.edu/castles/.); (4) Inada et al. 2007; (5) Kavo et al. 2010; (6) Inada et al. 2006a; (7) Sluse et al. 2008; (8) Oscoz et al. 1997; (9) Lubin et al. 2000; (9) Inada et al. 2003a; (10) Ofek et al. 2007; (11) Eigenbrod et al. 2006a; (12) Schechter et al. 1998; (13) Walsh et al. 1979; (14) Young et al. 1980; (15) Oguri et al. 2005; (16) Inada et al. 2003b; (17) Oguri et al. 2004b; (18) Inada et al. 2005a; (19) Inada et al. 2008b; (20) Pindor et al. 2006; (21) Wevmann et al. 1980; (22) Kundic et al. 1997; (23) Oguri et al. 2008b; (24) Paper II (25) Eigenbrod et al. 2006b; (26) Kavo et al. 2007; (27) Ofek et al. 2007; (28) C. E. Rusu et al., in preparation; (29) Oguri et al. 2008d; (30) Morokuma et al. 2007; (31) Oguri et al. 2004a; (32) Inada et al., in preparation; (33) Jackson et al. 2012. 
Table 6. DR7 Additional Lensed Quasars

\begin{tabular}{|c|c|c|c|c|c|c|c|c|c|c|c|}
\hline Object & $N_{\text {img }}$ & $z_{s}{ }^{\mathrm{a}}$ & $z_{l}^{\mathrm{b}}$ & $i^{\mathrm{c}}$ & $\theta^{\mathrm{d}}$ & $f_{I} \mathrm{e}$ & $I_{\text {gal }} \mathrm{f}$ & Source & Non-SDSS designation & Reject $^{\mathrm{g}}$ & Ref. \\
\hline SDSS J013435.66-093102.9 & 5 & 2.220 & 0.765 & 19.62 & 0.68 & 0.03 & $19.27(0.08)$ & DR3 & PMN J0134-0931 & $z>2.2, i>19.1, \theta<1^{\prime \prime}$ & $1,2,3,4$ \\
\hline SDSS J014516.59-094517.3 & 2 & 2.730 & 0.491 & 16.57 & 2.23 & 0.15 & $18.72(0.03)$ & DR3 & Q0142-100 & $z>2.2,|\Delta I|>1.25$ & $4,5,6$ \\
\hline SDSS J074352.61+245743.6 & 2 & 2.165 & 0.381 & 19.01 & 1.03 & 0.26 & $20.47(0.37)$ & DR3 & ULAS J0743+2457 & $|\Delta I|>1.25$ & 7,8 \\
\hline SDSS J081331.28+254503.0 & 4 & 1.510 & $\sim 0.1$ & 15.30 & 0.91 & 0.06 & $16.62(0.81)$ & DR3 & $\mathrm{HS} 0810+2554$ & $\theta<1^{\prime \prime}$ & 4,9 \\
\hline SDSS J081959.80+535624.2 & 2 & 2.239 & 0.294 & 18.52 & 4.04 & 0.23 & $16.54(0.04)$ & DR5 & $\ldots$ & $z>2.2,|\Delta I|>1.25$ & 10 \\
\hline SDSS J082016.11+081215.9 & 2 & 2.024 & 0.803 & 19.05 & 2.20 & 0.12 & $19.59(0.06)$ & DR5 & ULAS J0820+0812 & $|\Delta I|>1.25$ & 11 \\
\hline SDSS J082138.94+121729.9 & 2 & 3.113 & 0.39 & 18.76 & 2.56 & 0.14 & $19.05(0.22)$ & DR7 & HS0818+1227 & $z>2.2,|\Delta I|>1.25$ & 4,12 \\
\hline SDSS J083216.99+040405.2 & 2 & 1.116 & 0.659 & 18.89 & 1.98 & 0.22 & $19.35(0.03)$ & DR3 & $\cdots$ & $|\Delta I|>1.25$ & 13 \\
\hline SDSS J090334.94+502819.3 & 2 & 3.584 & 0.388 & 19.50 & 2.84 & 0.46 & $19.91(0.01)$ & DR3 & $\ldots$ & $z>2.2, i>19.1$ & 4,14 \\
\hline SDSS J090404.15+151254.5 & 2 & 1.826 & $\sim 0.3$ & 17.51 & 1.13 & 0.27 & $18.60(0.07)$ & DR7 & $\ldots$ & $|\Delta I|>1.25$ & 15 \\
\hline SDSS J091127.61+055054.0 & 4 & 2.763 & 0.769 & 17.78 & 3.25 & 0.31 & $20.43(0.02)$ & DR3 & RX J0911+0551 & $z>2.2$ & $4,16,17$ \\
\hline SDSS J094604.79+183539.7 & 2 & 4.799 & 0.388 & 18.74 & 3.06 & 0.91 & $17.61(0.02)$ & DR7 & $\ldots$ & $z>2.2$ & 18 \\
\hline SDSS J100424.88+122922.2 & 2 & 2.640 & 0.95 & 20.14 & 1.54 & 0.09 & $21.64(0.05)$ & DR5 & $\mathrm{J} 1004+1229$ & $z>2.2, i>19.1,|\Delta I|>1.25$ & 4,19 \\
\hline SDSS J102913.94+262317.9 & 3 & 2.199 & 0.58 & 18.55 & 22.54 & 0.95 & $19.26(0.02)$ & DR7 & $\ldots$ & $\theta>20^{\prime \prime}$ & 20,21 \\
\hline SDSS J105440.83+273306.4 & 2 & 1.452 & $\sim 0.2$ & 16.81 & 1.27 & 0.18 & $18.75(0.05)$ & DR7 & $\ldots$ & $|\Delta I|>1.25$ & $15^{\mid}$ \\
\hline SDSS J112818.49+240217.4 & 2 & 1.608 & $\sim 0.2$ & 17.89 & 0.84 & 0.54 & $18.56(0.06)$ & DR7 & $\ldots$ & $\theta<1^{\prime \prime}$ & 7 ڤ \\
\hline SDSS J113157.72+191527.7 & 2 & 2.915 & $\sim 0.3$ & 18.00 & 1.46 & 0.21 & $19.45(0.07)$ & DR7 & $\ldots$ & $z>2.2,|\Delta I|>1.25$ & 15 \\
\hline SDSS J113803.73+031457.8 & 4 & 2.443 & 0.445 & 19.00 & 1.44 & 0.35 & $20.03(0.01)$ & DR3 & $\ldots$ & $z>2.2$ & $4,22,23$ \\
\hline SDSS J115517.34+634622.0 & 2 & 2.888 & 0.176 & 17.68 & 1.95 & 0.50 & $17.81(0.16)$ & DR3 & $\ldots$ & $z>2.2$ & 4,24 \\
\hline SDSS J115518.29+193942.2 & 2 & 1.019 & 0.439 & 16.56 & 1.56 & 0.05 & $19.25(0.02)$ & DR7 & B1152+199 & $|\Delta I|>1.25$ & 4,25 \\
\hline SDSS J125418.94+223536.5 & 2 & 3.626 & $\sim 0.3$ & 19.20 & 1.56 & 0.57 & $20.18(0.04)$ & DR7 & $\ldots$ & $z>2.2, i>19.1$ & 10 \\
\hline SDSS J125819.24+165717.6 & 2 & 2.702 & 0.505 & 18.75 & 1.28 & 0.67 & $19.23(0.05)$ & DR7 & $\ldots$ & $z>2.2$ & 10 \\
\hline SDSS J130443.58+200104.2 & 2 & 2.175 & $0.373 ?$ & 18.45 & 1.87 & 0.24 & $18.74(0.08)$ & DR7 & $\ldots$ & $|\Delta I|>1.25$ & 15 \\
\hline SDSS J132236.41+105239.4 & 2 & 1.717 & $\sim 0.55$ & 18.23 & 2.00 & 0.21 & $19.67(0.03)$ & DR5 & $\ldots$ & $|\Delta I|>1.25$ & 15 \\
\hline SDSS J133401.39+331534.3 & 2 & 2.426 & $(0.557)$ & 18.78 & 0.83 & 0.73 & $21.50(0.37)$ & DR7 & $\ldots$ & $\theta<1^{\prime \prime}$ & 26 \\
\hline SDSS J133907.13+131039.6 & 2 & 2.241 & $\sim 0.4$ & 18.72 & 1.69 & 0.85 & $19.25(0.05)$ & DR7 & $\ldots$ & $z>2.2$ & 10 \\
\hline SDSS J134929.84+122706.8 & 2 & 1.722 & $\sim 0.65$ & 17.44 & 3.00 & 0.29 & $19.35(0.05)$ & DR5 & $\ldots$ & $|\Delta I|>1.25$ & 15 \\
\hline SDSS J140012.77+313454.1 & 2 & 3.317 & $\sim 0.8$ & 19.92 & 1.74 & 0.63 & $20.01(0.04)$ & DR7 & $\ldots$ & $z>2.2, i>19.1$ & 10 \\
\hline SDSS J140624.82+612640.9 & 2 & 2.135 & 0.271 & 19.31 & 1.98 & 0.58 & $18.12(0.02)$ & DR3 & $\ldots$ & $i>19.1$ & 27 \\
\hline SDSS J141546.24+112943.4 & 4 & 2.560 & $\sim 1.9$ & 16.91 & 1.35 & 0.70 & $\ldots$ & DR7 & $\mathrm{H} 1413+117$ & $z>2.2$ & $4,28,29$ \\
\hline SDSS J152720.13+014139.6 & 2 & 1.439 & $\sim 0.3$ & 18.30 & 2.58 & 0.11 & $19.29(0.18)$ & DR7 & ULAS J1527+0141 & $|\Delta I|>1.25$ & 8 \\
\hline SDSS J152938.90+103803.9 & 2 & 1.971 & $\sim 0.4$ & 17.58 & 1.27 & 0.19 & $19.62(0.40)$ & DR7 & ULAS J1529+1038 & $|\Delta I|>1.25$ & 8 \\
\hline SDSS J162026.14+120342.0 & 2 & 1.158 & 0.398 & 19.10 & 2.77 & 0.30 & $17.59(0.45)$ & DR7 & $\ldots$ & $|\Delta I|>1.25$ & 15 \\
\hline SDSS J163348.98+313411.9 & 2 & 1.518 & $(0.684)$ & 16.88 & 0.66 & 0.27 & $18.24(0.05)$ & DR3 & FBQ $1633+3134$ & $\theta<1^{\prime \prime},|\Delta I|>1.25$ & 4,30 \\
\hline SDSS J165043.44+425149.3 & 2 & 1.543 & $(0.577)$ & 17.32 & 1.18 & 0.17 & $20.50(0.10)$ & DR3 & $\ldots$ & $|\Delta I|>1.25$ & 4,31 \\
\hline
\end{tabular}


Table 6-Continued

\begin{tabular}{|c|c|c|c|c|c|c|c|c|c|c|c|}
\hline Object & $N_{\text {img }}$ & $z_{s}^{\mathrm{a}}$ & $z_{l}^{\mathrm{b}}$ & $i^{\mathrm{c}}$ & $\theta^{\mathrm{d}}$ & $f_{I} \mathrm{e}$ & $I_{\text {gal }} \mathrm{f}$ & Source & Non-SDSS designation & Reject $^{\mathrm{g}}$ & Ref. \\
\hline SDSS J234311.94-005034.3 & 2 & 0.787 & $\sim 0.3$ & 20.09 & 1.51 & 0.85 & $19.16(0.10)$ & DR5 & ULAS J2343-0050 & $i>19.1$ & 32 \\
\hline
\end{tabular}

Note. - See text for the selection of each lensed quasar.

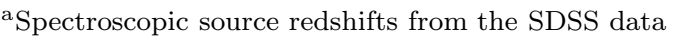

b Lens redshifts. Values in parentheses indicate lens redshifts measured from absorption lines in the quasar spectra rather than direct spectroscopy of the lensing galaxy. For lenses without any spectroscopic information, we provide an approximate redshift with an error-bar inferred from the color and magnitude.

cSDSS PSF magnitude in $i$-band (AB) after correcting for the Galactic extinction.

${ }^{\mathrm{d}}$ Maximum image separations, in units of arcsec, derived from the best imaging data

${ }^{\mathrm{e}} I$-band flux ratios between the brightest lensed image and the farthest lensed image from the brightest image.

${ }^{\mathrm{f}} I$-band (Vega) magnitude of the lensing galaxy without Galactic extinction corrections. Values in parentheses are statistical errors. When the system contains multiple galaxies within the Einstein radius, the magnitude of the brightest galaxy is shown.

${ }^{\mathrm{g}}$ The reason that each lens is excluded from the statistical sample.

References. - (1) Winn et al. 2002; (2) Gregg et al. 2002; (3) Hall et al. 2002; (4) CASTLES webpage (C. S. Kochanek et al., http://cfa-www.harvard.edu/castles/ ). (5) Surdej et al.|1987; (6) Eigenbrod et al.|2007; (7) Inada et al., in preparation; (8) Jackson et al. 2012; (9) Reimers et al. 2002; (10) Inada et al. 2009; (11) Jackson et al. 2009; (12) Hagen \& Reimers 2000; (13) Oguri et al. 2008b; (14) Johnston et al. 2003; (15) Kavo et al. 2010; (16) Bade et al. 1997; (17) Kneib et al. 2000; (18) McGreer et al. 2010; (19) Lacv et al. 2002; (20) Inada et al. 2006b; (21) Oguri et al. 2008c; (22) Paper II; (23) Eigenbrod et al. 2006b; (24) Pindor et al. 2004; (25) Mvers et al.|1999; (26) Rusu et al. 2011; (27) Inada et al. 2007; (28) Magain et al. 1988; (29) Goicoechea \& Shalvapin 2010; (30) Morgan et al. 2001; (31) Morgan et al. 2003; (32) Jackson et al. 2008. 

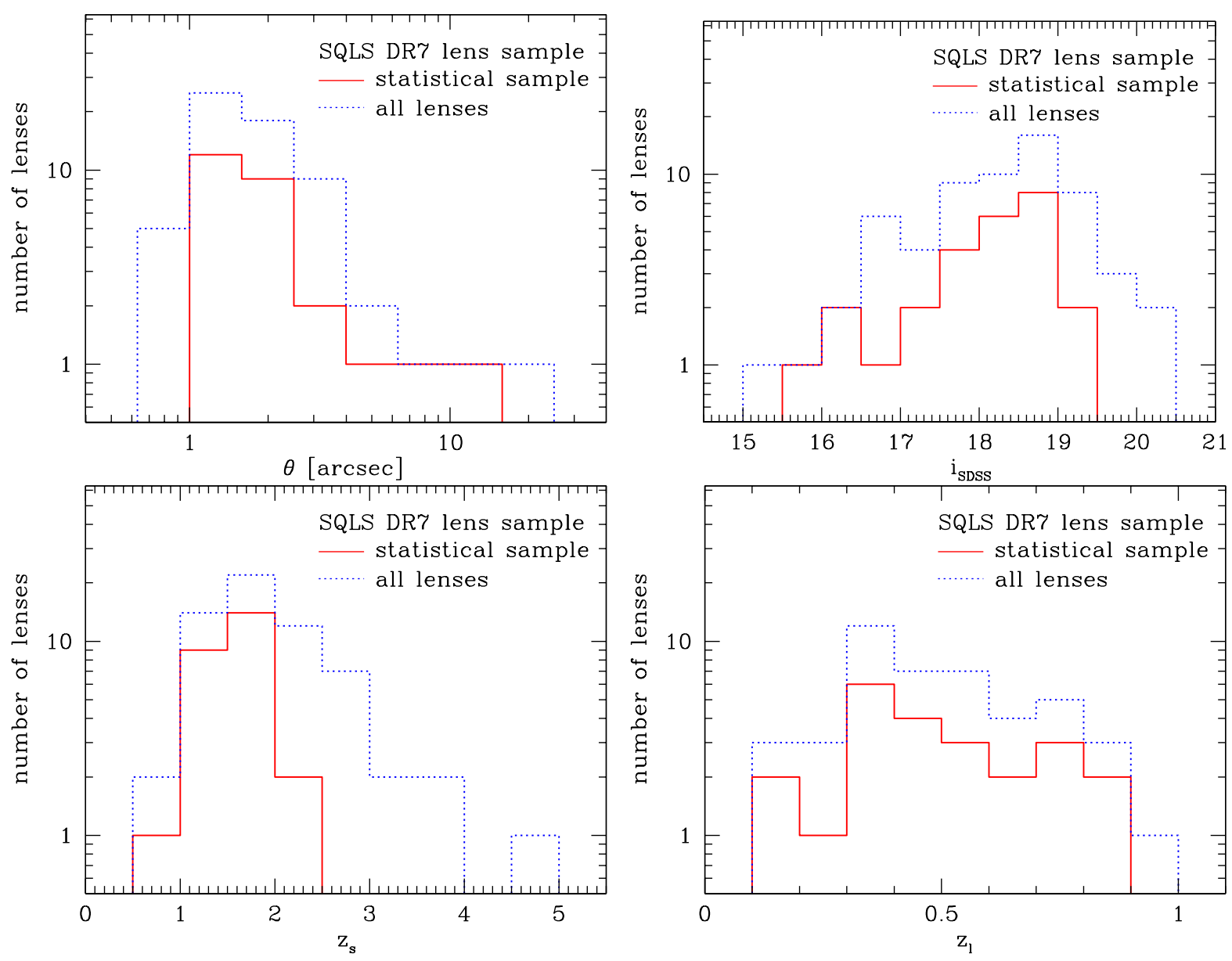

Fig. 1.- Properties of the SQLS lenses in the statistical sample (solid) and all lenses (dotted). Distributions of the image separations $\theta$ (upper left), the $i$-band PSF magnitudes $i_{\text {SDSS }}$ (upper right), the source redshifts $z_{s}$ (lower left), and the lens redshifts $z_{l}$ (lower right) are shown. For the lens redshift distribution, we use only the lens systems whose lens redshifts are measured spectroscopically (including those measured by absorption lines in the quasar spectra). 

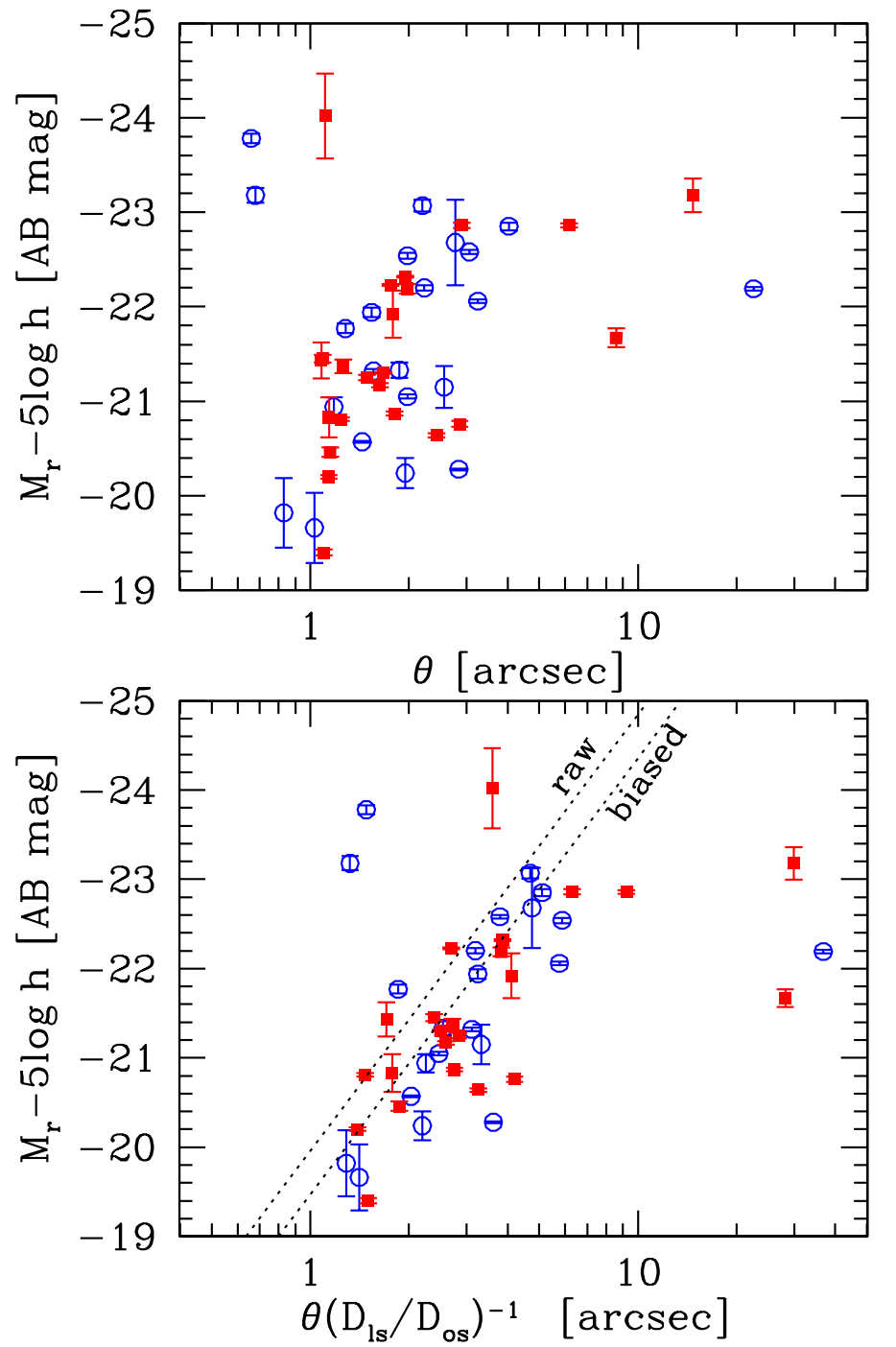

Fig. 2.- The correlation between the image separation $\theta$ and the absolute $r$-band magnitude of the lens galaxy $M_{r}$. The absolute magnitudes are computed from the observed $I$-band magnitudes listed in Tables 5 and 6. A template spectrum of an elliptical galaxy by Coleman et al. (1980) is used to compute the cross-filter $K$-correction. Filled squares show lenses in the statistical sample and open circles are additional lenses. Error-bars show only the statistical errors of the apparent magnitude measurements. While the raw image separations are used in the upper panel, in the lower panel we rescale the image separation with the distance ratio $\left(D_{l s} / D_{o s}\right)^{-1}$ in order to eliminate the redshift dependence of the image separation. The dotted lines show the predicted correlation from the Faber-Jackson relation measured in the SDSS data with the luminosity evolution extrapolated to $z=0.5$ (Bernardi et al. 2003) with and without the effect of the lensing selection bias (see text for details). 